\title{
Neutrons and magnetism
}

\author{
Mechthild Enderle \\ Institut Laue-Langevin, 6, Rue Jules Horowitz, BP. 156, 38042 Grenoble Cedex, France
}

\begin{abstract}
Neutron scattering is a unique technique in magnetism, since it measures directly the Fourier transform of the time-dependent magnetic pair correlations. The neutron interacts only weakly with matter, so that each neutron normally only scatters once in the sample volume. The dynamic pair correlation functions are thus probed in the whole volume of the sample. Here we summarize the relevant formalism and approximations for neutron scattering on magnetic materials, focusing on the investigation of single crystals. The lecture aims to stimulate - not to replace - the study of the relevant literature [1-9].
\end{abstract}

\section{THERMAL NEUTRON SCATTERING}

\subsection{Properties of the neutron}

We recall some important properties of the neutron:

- wave (interference, even with itself)

- particle (mass $m$, energy-momentum relation, interactions)

- lifetime of free neutron: 15 min (allows to do experiments)

- momentum $\boldsymbol{p}=\hbar \boldsymbol{k}=\hbar \frac{2 \pi}{2}$

- energy $E=\frac{p^{2}}{2 m}=\frac{\hbar^{2}}{2 m} k^{2}=2.072 k^{2} \mathrm{meV}^{2}$

- no charge

- spin $1 / 2$ and magnetic moment $\mu_{n}=-\gamma \mu_{N} \hat{\boldsymbol{\sigma}}$, where $\gamma=1.913, \mu_{N}=\frac{e \hbar}{2 m_{p}}$ is the nuclear magneton, and $\hat{\boldsymbol{\sigma}}$ is the Pauli spin operator.

\subsection{Master equation}

All interactions of thermal (slow) neutrons with matter are weak, either due to the weakness of the interaction itself (interaction with the unpaired electrons, Foldy, spin-orbit), or, as in the case of the strong interaction with the nucleus, due to the extreme short-range character of the interaction and the extreme "dilution" of the point-like scattering nuclei in the scattering volume. The neutron therefore probes the whole volume, and generally each neutron scatters only once within the sample volume. Further, the scattering process can be treated in first order perturbation, the first Born approximation

This is an Open Access article distributed under the terms of the Creative Commons Attribution License 4.0, which permits unrestricted use, distribution, and reproduction in any medium, provided the original work is properly cited. 


\section{Collection SFN}

is valid, and the differential cross section can be calculated according to Fermi's golden rule, with the scattering amplitude given by the transition matrix element of the interaction potential (neutron properties are colored blue, sample properties red, and the interaction green):

$$
\begin{gathered}
\left.\frac{d^{2} \sigma}{d \Omega d E_{f}}\right|_{n_{0}, \sigma_{i} \rightarrow n_{1}, \sigma_{f}}=\frac{k_{f}}{k_{i}}\left(\frac{m}{2 \pi \hbar^{2}}\right)^{2}\left|\left\langle\boldsymbol{k}_{f} \sigma_{f} n_{1}|V| \boldsymbol{k}_{i} \sigma_{i} n_{0}\right\rangle\right|^{2} \delta\left(\epsilon_{1}-\epsilon_{0}-\left(E_{i}-E_{f}\right)\right) \\
\frac{d^{2} \sigma}{d \Omega d E_{f}} \\
=\frac{k_{f}}{k_{i}}\left(\frac{m}{2 \pi \hbar^{2}}\right)^{2} \sum_{\substack{\sigma_{i}, \sigma_{f} \\
n_{0}, n_{1}}} p\left(\sigma_{i}\right) p\left(n_{0}\right)\left|\left\langle\boldsymbol{k}_{f} \sigma_{f} n_{1}|V| \boldsymbol{k}_{i} \sigma_{i} n_{0}\right\rangle\right|^{2} \delta\left(\epsilon_{1}-\epsilon_{0}-\hbar \omega\right),
\end{gathered}
$$

where
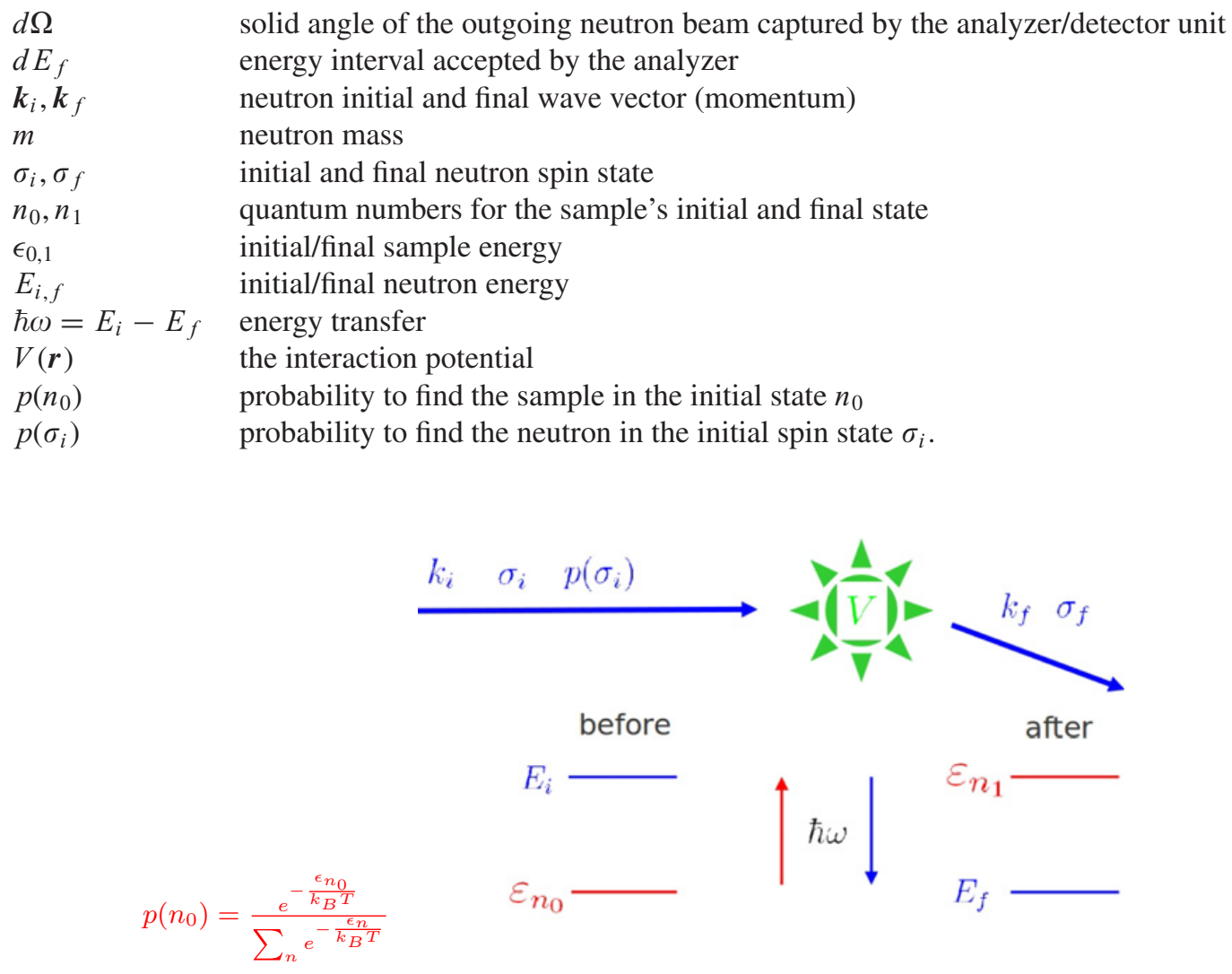

The neutron beam is generated and analyzed far from the sample, so that the incoming neutron state can be assumed as a plane wave state $e^{i \boldsymbol{k}_{i} \boldsymbol{r}}$. The near-point-like scatterer(s) emit spherical wave(s) and are detected far away, so that their interference, the outgoing neutron wave, again can be treated as plane wave. The matrix element can therefore be rewritten as space Fourier transform of the interaction potential, taken at the momentum transfer $\boldsymbol{Q}=\boldsymbol{k}_{i}-\boldsymbol{k}_{f}$. (This is valid for an interaction potential that commutes with $\boldsymbol{Q} \cdot \boldsymbol{r}$.) Thermal neutron energies are for most isotopes far away from nuclear resonances (with few exceptions) so that absorption often can be neglected. 
scattering length

$\begin{array}{llll}\text { nucleus } & +6.5 & \mathrm{fm} & \\ \text { electronic magn. mom. } & -5.4 & \mathrm{fm} & (\cdot S(J)) \\ \text { electric field } & +1.5 & \mathrm{am} & \end{array}$

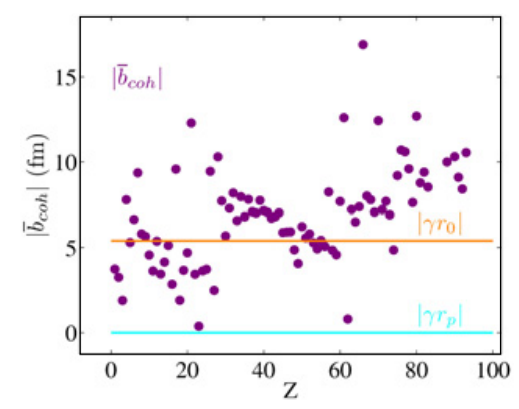

Figure 1. Average absolute coherent nuclear scattering length in comparison to the "magnetic scattering length" $\gamma r_{0}$ of a spin 1 and to the "electric scattering length" $\gamma r_{p}$ of one elementary charge. The coherent nuclear scattering length can have positive or negative sign and depends on the isotope, there is no systematic variation with the atomic number $Z . r_{0}=\frac{\mu_{0}}{4 \pi} \frac{e^{2}}{m_{e}}$ is the first Bohr radius, and $r_{p}$ the corresponding quantity replacing the electron mass $m_{e}$ by the proton mass $m_{p}$.

\subsection{Types of interaction}

The neutron interacts with matter via different forces:

$$
\begin{aligned}
& \text { neutron }- \text { atomic nucleus } \\
& \text { neutron }- \text { electronic magnetic moment } \\
& \text { neutron }- \text { electric field }
\end{aligned}
$$

\author{
strong interaction \\ dipole-dipole interaction \\ spin-orbit + Foldy interaction
}

The interaction with the electronic magnetic moment is of the same order of magnitude as the interaction with the atomic nuclei - this is one of the reasons why neutron scattering is such a powerful technique for magnetism. The "scattering length" corresponding to the interaction with electric fields is about three orders of magnitude weaker, i.e. the corresponding cross section is six orders of magnitude smaller.

\subsection{Nuclear interaction potential}

The Fermi pseudopotential $V_{N}$ for neutron scattering from one nucleus can be written as

$$
\begin{aligned}
V_{N}(\boldsymbol{r}) & =\frac{2 \pi \hbar^{2}}{m} b \delta\left(\boldsymbol{r}-\boldsymbol{R}_{j}\right) \\
b & =A(I)+B(I) \hat{\sigma} \cdot \hat{\boldsymbol{I}}
\end{aligned}
$$

the scattering length $b$ depends on

- the isotope

- the nuclear spin value $I$

- the total spin of neutron+nucleus: $b^{ \pm}$if the total spin is $I \pm \frac{1}{2}$.

The mean value $\bar{b}$ (average over different isotopes and nuclear spin states) for a given crystal site leads to interference between the scattered waves from different sites and thus coherent scattering (nuclear Bragg peaks, phonons), the standard deviation from the mean value, $\sqrt{\overline{\left(b^{2}\right)}-(\bar{b})^{2}}$, leads to incoherent scattering. 


\section{Collection SFN}

\subsection{Magnetic interaction potential}

The magnetic potential $V_{M}$ for the scattering of one neutron with magnetic moment $\boldsymbol{\mu}_{n}$ from the magnetic field $\boldsymbol{B}_{e}(\boldsymbol{r})$ of one electron is

$$
V_{M}(\boldsymbol{r})=-\boldsymbol{\mu}_{n} \cdot \boldsymbol{B}_{e}(\boldsymbol{r})
$$

Maxwell equation: $\nabla \cdot \boldsymbol{B}_{e}(\boldsymbol{r})=0$

Contract $e^{-i \boldsymbol{k}_{f} \boldsymbol{r}}$ and $e^{i \boldsymbol{k}_{i} \boldsymbol{r}}$ to $e^{i \boldsymbol{Q} \boldsymbol{r}}$ and Fourier transform the interaction

$$
\left\langle\boldsymbol{k}_{f} \sigma_{f} n_{1}\left|-\boldsymbol{\mu}_{n} \cdot \boldsymbol{B}_{e}(\boldsymbol{r})\right| \boldsymbol{k}_{i} \sigma_{i} n_{0}\right\rangle=\left\langle\sigma_{f} n_{1}\left|-\boldsymbol{\mu}_{n} \cdot \boldsymbol{B}_{e}(\boldsymbol{Q})\right| \sigma_{i} n_{0}\right\rangle
$$

Fourier transformed Maxwell equation

$$
\boldsymbol{Q} \cdot \boldsymbol{B}_{e}(\boldsymbol{Q})=0 .
$$

The divergence-free magnetic field of the electron is hence always perpendicular to the momentum transfer. In consequence, the neutron "feels" only that part of the electronic magnetic field (coming from both spin and orbital moment) which is perpendicular to the momentum transfer [14]. This will provide us with an easy-to-remember motivation of the so-called "magnetic selection rule". To obtain the relation between magnetic moment direction and momentum transfer, we write the magnetic field $\boldsymbol{B}_{e}=\nabla \times \boldsymbol{A}$ of an electron via the vector potential $\boldsymbol{A}=\nabla \times \frac{\boldsymbol{\mu}_{e}^{t o t}}{r}$, where $\boldsymbol{\mu}_{e}^{t o t}=\boldsymbol{\mu}_{e}^{S}+\boldsymbol{\mu}_{e}^{L}$. The interaction potential becomes

vector potential $\boldsymbol{A}$

$$
\begin{aligned}
V_{M}(r) & =-\boldsymbol{\mu}_{n} \cdot \nabla \times \overbrace{\text { Spin }}^{\nabla \times \frac{\boldsymbol{\mu}_{e}^{S}}{r}}+\underbrace{\nabla \times \frac{\boldsymbol{\mu}_{e}^{L}}{r}}_{\text {orbital current }}) \\
& =\underbrace{\frac{\boldsymbol{\mu}_{n}\left(\boldsymbol{\mu}_{e}^{S}+\boldsymbol{\mu}_{e}^{L}\right)}{r^{3}}-\frac{3\left(\boldsymbol{\mu}_{n} \cdot \boldsymbol{r}\right)\left(\left(\boldsymbol{\mu}_{e}^{S}+\boldsymbol{\mu}_{e}^{L}\right) \cdot \boldsymbol{r}\right)}{r^{5}}}_{\text {dipole-dipole interaction }}-\underbrace{\frac{8 \pi}{3} \boldsymbol{\mu}_{n}\left(\boldsymbol{\mu}_{e}^{S}+\boldsymbol{\mu}_{e}^{L}\right) \delta(\boldsymbol{r}) .}_{\text {contact term }}
\end{aligned}
$$

The contact term describes the interaction when neutron and electron are at exactly the same place, which is possible since they are distinguishable particles.

We consider the magnetic matrix element of one electron which is proportional to

$$
\left\langle\boldsymbol{k}_{f} \sigma_{f} n_{1}\left|-\boldsymbol{\mu}_{n} \cdot \nabla \times\left(\nabla \times \frac{\boldsymbol{\mu}_{e}^{t o t}}{r}\right)\right| \boldsymbol{k}_{i} \sigma_{i} n_{0}\right\rangle .
$$

The next step, "contract the plane wave states and take the Fourier transform of the interaction potential" is not obvious, derivations can be found in $[1,2,4]$. A crucial point is that $V_{M}$ commutes with $\boldsymbol{Q} \cdot \boldsymbol{r}$ in spite of the explicit dependence of $V_{M}$ on the momentum $\boldsymbol{p}$ of the electron (via $\mu_{e}^{L}$ ) [1]. After the Fourier transform, " $\nabla \times$ " becomes essentially " $-i \boldsymbol{Q} \times$ " (cf. [1]) and we obtain

$$
\begin{aligned}
\left\langle\sigma_{f} n_{1}\left|\boldsymbol{\mu}_{n} \cdot\left(\hat{\boldsymbol{Q}} \times\left(\hat{\boldsymbol{Q}} \times \boldsymbol{\mu}_{e}^{t o t}(\boldsymbol{Q})\right)\right)\right| \sigma_{i} n_{0}\right\rangle & =\left\langle\sigma_{f} n_{1}|-\boldsymbol{\mu}_{n} \cdot \underbrace{\left(\hat{\boldsymbol{Q}} \times\left(\boldsymbol{\mu}_{e}^{t o t}(\boldsymbol{Q}) \times \hat{\boldsymbol{Q}}\right)\right)}| \sigma_{i} n_{0}\right\rangle \\
& =\left\langle\sigma_{f} n_{1}\left|\quad-\boldsymbol{\mu}_{n} \cdot \boldsymbol{\mu}_{e}^{t o t}(\boldsymbol{Q}) \quad\right| \sigma_{i} n_{0}\right\rangle \\
& \propto\left\langle\sigma_{f} n_{1}\left|-\sigma \cdot \boldsymbol{\mu}_{e}{ }^{t o t}(\boldsymbol{Q})\right| \sigma_{i} n_{0}\right\rangle .
\end{aligned}
$$


For the entire sample we have

$$
\sum \boldsymbol{\mu}_{e \perp}^{t o t}(\boldsymbol{Q})=\boldsymbol{M}_{\perp}(\boldsymbol{Q}) \text {. }
$$

This leads to the magnetic cross section

$$
\begin{aligned}
\frac{d^{2} \sigma}{d \Omega d E_{f}}= & \frac{k_{f}}{k_{i}}\left(\frac{m}{2 \pi \hbar^{2}}\right)^{2} \sum_{\substack{\sigma_{i}, \sigma_{f} \\
n_{0}, n_{1}}} p\left(\sigma_{i}\right) p\left(n_{0}\right)\left|\left\langle\boldsymbol{k}_{f} \sigma_{f} n_{1}\left|V_{M}\right| \boldsymbol{k}_{i} \sigma_{i} n_{0}\right\rangle\right|^{2} \delta\left(\epsilon_{1}-\epsilon_{0}-\hbar \omega\right) \\
= & \frac{k_{f}}{k_{i}}\left(\gamma r_{0}\right)^{2} \sum_{\substack{\sigma_{i}, \sigma_{f} \\
n_{0}, n_{1}}} p\left(\sigma_{i}\right) p\left(n_{0}\right)\left|\left\langle\sigma_{f} n_{1}\left|\sigma \cdot \boldsymbol{M}_{\perp}(\boldsymbol{Q})\right| \sigma_{i} n_{0}\right\rangle\right|^{2} \delta\left(\epsilon_{1}-\epsilon_{0}-\hbar \omega\right)
\end{aligned}
$$

and to the "magnetic selection rule"

$$
M_{\perp}(Q)=\hat{Q} \times(M(Q) \times \hat{Q})
$$

where we have the Fourier transform of the magnetization (operator) density

$$
\boldsymbol{M}(\boldsymbol{Q})=\int d^{3} r e^{i \boldsymbol{Q} r} \boldsymbol{M}(\boldsymbol{r}) .
$$

Note that since $\boldsymbol{M}(\boldsymbol{Q})$ is an operator acting on the sample states, the matrix element of $\boldsymbol{M}(\boldsymbol{Q})$ for $n_{1}=n_{0}(\omega=0)$ corresponds to the Fourier transformed static magnetization density, while for $n_{1} \neq n_{0}$ $(\omega \neq 0)$, it may e.g. correspond to the eigenvector of a spin-wave excitation.

For unpolarized incoming neutrons and no polarization analysis the sum over the neutron spin states is straightforward. We also sum over the (complete set of) final sample energy states, rewrite the $\delta$-function as integral and write the magnetization operators in the Heisenberg picture. The magnetic cross section then reads

$$
\begin{aligned}
& \frac{d^{2} \sigma}{d \Omega d E_{f}}=\frac{k_{f}}{k_{i}}\left(\gamma r_{0}\right)^{2} \sum_{\sigma_{i}, \sigma_{f}, n_{0}, n_{1}} p\left(\sigma_{i}\right) p\left(n_{0}\right)\left|\left\langle\sigma_{f} n_{1}\left|\sigma \cdot \boldsymbol{M}_{\perp}(\boldsymbol{Q})\right| \sigma_{i} n_{0}\right\rangle\right|^{2} \delta\left(\epsilon_{1}-\epsilon_{0}-\hbar \omega\right) \\
& =\frac{k_{f}}{k_{i}}\left(\gamma r_{0}\right)^{2} \underbrace{\sum_{n_{0}} p\left(n_{0}\right) \frac{1}{2 \pi \hbar} \int_{-\infty}^{\infty} d t e^{-i \omega t}\left\langle n_{0}\left|\boldsymbol{M}_{\perp}^{*}(\boldsymbol{Q}, 0) \cdot \boldsymbol{M}_{\perp}(\boldsymbol{Q}, t)\right| n_{0}\right\rangle} \\
& =\frac{k_{f}}{k_{i}}\left(\gamma r_{0}\right)^{2}\left\langle\boldsymbol{M}_{\perp}^{*}(\boldsymbol{Q}) \cdot \boldsymbol{M}_{\perp}(\boldsymbol{Q})\right\rangle_{T, \omega} \\
& \text { scattering function } S(\boldsymbol{Q}, \omega) \\
& =\frac{k_{f}}{k_{i}}\left(\gamma r_{0}\right)^{2} \sum_{\alpha \beta}\left(\delta_{\alpha \beta}-\hat{Q}^{\alpha} \hat{Q}^{\beta}\right) \underbrace{\left\langle M^{\alpha *}(\boldsymbol{Q}) M^{\beta}(\boldsymbol{Q})\right\rangle_{T, \omega}}_{S^{\alpha \beta}(\boldsymbol{Q}, \omega)}
\end{aligned}
$$

where we have defined a scattering function, that we will now consider in more detail. 


\section{Collection SFN}

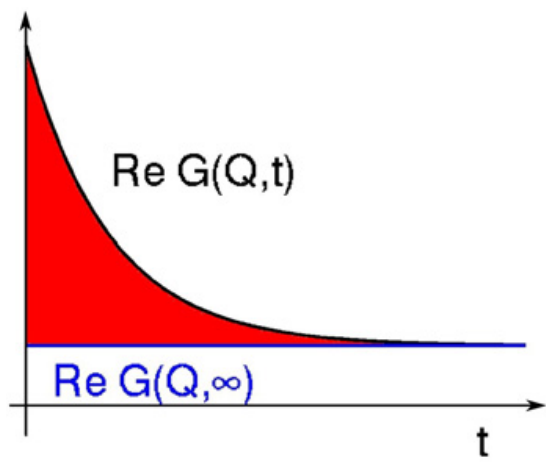

Figure 2. Magnetic pair correlation function $G(\boldsymbol{Q}, t)=\left\langle M^{\alpha}(-\boldsymbol{Q}, 0) M^{\beta}(\boldsymbol{Q}, t)\right\rangle_{T}$ split into a time-independent part (blue) and "the rest" (space between blue and black curves). Only the real part is shown.

\section{SCATTERING FUNCTION AND SUSCEPTIBILITY}

First we note that the scattering function is the space and time Fourier transformed time dependent magnetic pair correlation function:

$$
\begin{aligned}
S^{\alpha \beta}(\boldsymbol{Q}, \omega) & =\left\langle M^{\alpha *}(\boldsymbol{Q}) M^{\beta}(\boldsymbol{Q})\right\rangle_{T, \omega} \\
& =\frac{1}{2 \pi \hbar} \int d t e^{-i \omega t}\left\langle M^{\alpha}(-\boldsymbol{Q}, 0) M^{\beta}(\boldsymbol{Q}, t)\right\rangle_{T} \\
& =\frac{1}{2 \pi \hbar} \int d^{3} r d t e^{i(\boldsymbol{Q} r-\omega t)} \underbrace{\left\langle M^{\alpha}(0,0) M^{\beta}(\boldsymbol{r}, t)\right\rangle_{T}}_{\text {time dependent magnetic pair correlation function } G(\boldsymbol{r}, t)} \cdot
\end{aligned}
$$

Here, $\langle\ldots\rangle_{T, \omega}$ denotes the quantum mechanical and thermal average at a constant temperature $T$ for a given constant energy transfer $\hbar \omega$, and $\langle\ldots\rangle_{T}$ the thermal average at constant temperature.

Next we split the (space-)Fourier transformed pair correlation function $G(\boldsymbol{Q}, t)=$ $\left\langle M^{\alpha}(-\boldsymbol{Q}, 0) M^{\beta}(\boldsymbol{Q}, t)\right\rangle_{T}$ (black) into a time independent part (blue) and "the rest" (red), as sketched below. In this chapter the color blue marks a time-independent (static) quantity, and red a time-dependent quantity without the static part.

\section{1 $t \rightarrow \infty$ limit of pair correlations}

The time independent part of the pair correlations is obtained by taking the limit $t \rightarrow \infty$. We consider its Fourier transform:

$$
\begin{aligned}
S_{\text {static }}^{\alpha \beta}(\boldsymbol{Q}, \omega) & =\frac{1}{2 \pi \hbar} \int d t e^{-i \omega t} \lim _{t \rightarrow \infty}\left\langle M^{\alpha}(-\boldsymbol{Q}, 0) M^{\beta}(\boldsymbol{Q}, t)\right\rangle_{T} \\
& =\frac{1}{2 \pi \hbar} \int d t e^{-i \omega t}\left\langle M^{\alpha}(-\boldsymbol{Q}, 0) M^{\beta}(\boldsymbol{Q}, \infty)\right\rangle_{T}
\end{aligned}
$$


Since there are no correlated deviations from average at $t \rightarrow \infty$ :

$$
=\frac{1}{2 \pi \hbar} \int d t e^{-i \omega t}\left\langle M^{\alpha}(-\boldsymbol{Q}, 0)\right\rangle_{T}\left\langle M^{\beta}(\boldsymbol{Q}, \infty)\right\rangle_{T} .
$$

The thermal average of $\left\langle M^{\alpha}(\boldsymbol{Q}, t)\right\rangle_{T}$ is the same at $t \rightarrow \infty$ as at $t=0$ :

$$
\begin{aligned}
& =\frac{1}{2 \pi \hbar} \int d t e^{-i \omega t}\left\langle M^{\alpha}(-\boldsymbol{Q}, 0)\right\rangle_{T}\left\langle M^{\beta}(\boldsymbol{Q}, 0)\right\rangle_{T} \\
& =\delta(\omega)\left\langle M^{\alpha *}(\boldsymbol{Q}, 0)\right\rangle_{T}\left\langle M^{\beta}(\boldsymbol{Q}, 0)\right\rangle_{T}
\end{aligned}
$$

which gives the magnetic Bragg peak scattering at energy $\hbar \omega=0$, but also the purely elastic scattering in case of short range order like e.g. in spin glasses.

\subsection{Equal-time pair correlations: $t=0$}

Another important limit are the equal-time pair correlations, where $t=0$ in the pair correlation function. Their space Fourier transform is the energy integrated scattering function, which is called structure factor.

$$
\begin{aligned}
\left\langle M^{\alpha}(-\boldsymbol{Q}, 0) M^{\beta}(\boldsymbol{Q}, t=0)\right\rangle & =\int_{-\infty}^{+\infty} d \omega e^{-i \omega 0} S^{\alpha \beta}(\boldsymbol{Q}, \omega) \\
& =S^{\alpha \beta}(\boldsymbol{Q}, t=0)=S^{\alpha \beta}(\boldsymbol{Q}) .
\end{aligned}
$$

Experimentally, $S(\boldsymbol{Q})$ can be measured by explicit measurement of $S(\boldsymbol{Q}, \omega)$ at constant- $\boldsymbol{Q}$ and subsequent energy integration, e.g. on a time-of-flight (TOF) or triple-axis spectrometer (TAS). The integration interval needs not to be $[-\infty,+\infty]$, it is sufficient to cover the full energy range of the magnetic dynamics.

On diffraction instruments, where the final neutron energy is not analyzed (energy integration) and

$$
S(\boldsymbol{Q})=\int_{-\infty}^{+\infty} d \omega S(\boldsymbol{Q}, \omega)
$$

is approximated by

$$
\int_{0}^{E_{i}+k_{B} T} d E_{f} \frac{d \sigma}{d \Omega d E_{f}}=\int_{-k_{B} T}^{E_{i}} d \omega \frac{d \sigma}{d \Omega d E_{f}} \propto \int_{-k_{B} T}^{E_{i}} d \omega S(\psi, \phi, \omega) .
$$

This is valid if $E_{i} \gg \epsilon_{n}, \forall n$ for all excited magnetic states of the sample $\epsilon_{n}$. The incident neutron energy defines the upper integration limit (the neutron cannot give more energy to the sample than it has itself), the lower limit is approximately given by the temperature of the sample (the neutron cannot gain more energy from the sample than about $k_{B} T$, since the sample will not have any states thermally occupied above $k_{B} T$ ), and the scattering function $S(\boldsymbol{Q}, \omega)$ is zero for negative energies below $k_{B} T$ as well as above all excited states. Large enough $k_{i}\left(E_{i}\right)$ is important for yet another reason: Only for $E_{i} \gg \epsilon_{n} \forall n$, a fixed sample orientation $(\psi)$ and fixed $\boldsymbol{k}_{f}$-direction $(\phi)$ with variable length of $k_{f}$ correspond to approximately the same $\boldsymbol{Q}$, otherwise each final energy (and length of $k_{f}$ ) corresponds to another total momentum transfer $\boldsymbol{Q}$, see sketch below. 


\section{Collection SFN}

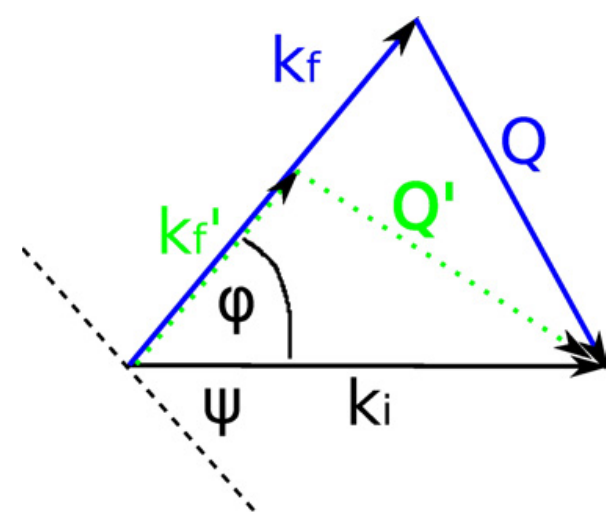

Figure 3. Scattering triangles for a fixed sample orientation $\psi$ and a fixed $\boldsymbol{k}_{f}$-direction $\phi$ at constant but too small incident neutron wave vector $\boldsymbol{k}_{i}$ : Different lengths of $k_{f}$ (blue solid and dashed green) correspond to different momentum transfers $\boldsymbol{Q}$ (blue solid and dashed green).

\subsection{Bragg and "diffuse" scattering}

We now consider what remains in the pair correlations in addition to the $t \rightarrow \infty$ limit:

$$
\begin{aligned}
& \left\langle M^{\alpha *}(\boldsymbol{Q}, 0) M^{\beta}(\boldsymbol{Q}, t)\right\rangle_{T} \\
= & \left.\left\langle M^{\alpha *}(\boldsymbol{Q}, 0)\right\rangle\left\langle M^{\beta}(\boldsymbol{Q}, 0)\right\rangle \ldots\right\} \rightarrow \text { Bragg, short range order, glassy frozen states: time independent } \\
& \ldots \underbrace{\left\langle\left(M^{\alpha *}(\boldsymbol{Q}, 0)-\left\langle M^{\alpha *}(\boldsymbol{Q}, 0)\right\rangle\right)\left(M^{\beta}(\boldsymbol{Q}, t)-\left\langle M^{\beta}(\boldsymbol{Q}, 0)\right\rangle\right)\right\rangle_{T}}_{\text {correlation of fluctuations }}
\end{aligned}
$$

and we find that after separation of all time-independent parts (Bragg, i.e. periodic long range order, short range order, even disordered frozen spin states), the "rest" corresponds to the correlations of the fluctuations (spin-waves and other magnetic excitations, paramagnetic scattering etc.). We call the Fourier transform of the latter "diffuse", it may nevertheless contain sharp delta-shaped excitations (cf. sketch below), and it may also be non-zero at $\omega=0$. The sketch below illustrates the relation between the time-independent and the diffuse ("rest") scattering, and their Fourier transforms which we call $S_{\text {static }}(\boldsymbol{Q}, \omega)$ and $S_{\text {diff }}(\boldsymbol{Q}, \omega)$, keeping in mind that "static" contains all time-independent scattering, even short-range order and glassy frozen states. (In some textbooks, contrary to here, all scattering which does not lead to Bragg peaks is called "diffuse", in others the entire time-independent part may be refered to as "Bragg" scattering.)

\subsection{Dynamic susceptibility}

The dynamic susceptibility is the linear response to a space and time varying magnetic field, defined via the expectation value of the magnetization density (cf. [3]):

$$
\left\langle M^{\alpha}(\boldsymbol{r}, t)\right\rangle_{T}=\left.\left\langle M^{\alpha}(\boldsymbol{r}, t)\right\rangle_{T}\right|_{H=0}+\sum_{\beta} \int d^{3} r^{\prime} d t^{\prime} \chi^{\alpha \beta}\left(\boldsymbol{r}-\boldsymbol{r}^{\prime}, t-t^{\prime}\right) H^{\beta}\left(\boldsymbol{r}^{\prime}, t^{\prime}\right)
$$



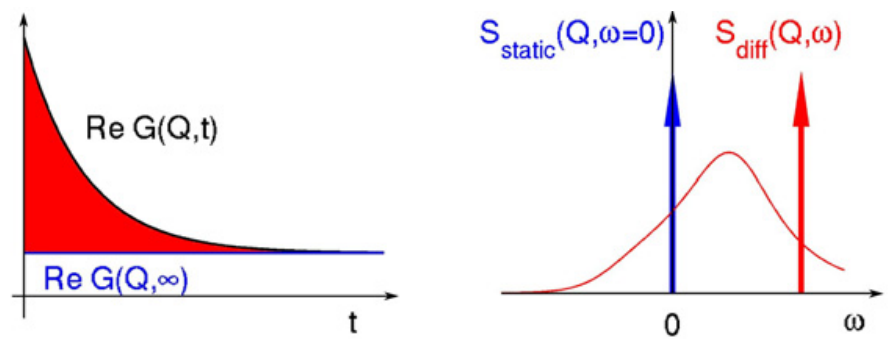

$$
S^{\alpha \beta}(\boldsymbol{Q}, \omega)=S_{\text {static }}^{\alpha \beta}(\boldsymbol{Q}, \omega=0)+S_{\text {diff }}^{\alpha \beta}(\boldsymbol{Q}, \omega)
$$

Figure 4. Sketch of the time-independent and time-dependent pair correlations (here shown as real), and their Fourier transforms, the static and "diffuse" scattering functions. Two different examples of diffuse scattering are displayed.

Fourier transform

$$
\left\langle M^{\alpha}(\boldsymbol{Q}, \omega)\right\rangle_{T}=\left.\left\langle M^{\alpha}(\boldsymbol{Q}, \omega)\right\rangle_{T}\right|_{H=0}+\sum_{\beta} \chi^{\alpha \beta}(\boldsymbol{Q}, \omega) H^{\beta}(\boldsymbol{Q}, \omega)
$$

$\left.\left\langle M^{\alpha}(\boldsymbol{Q}, \omega)\right\rangle_{T}\right|_{H=0}$ contains the Fourier transform of the field independent magnetization density, it will be a $\delta$-peak at zero energy (dynamic contributions cancel each other in the ensemble average). Before we look closer at the dynamic susceptibility, we consider which susceptibility we measure with a SQUID. The SQUID measures with a field at zero frequency (static), the magnetization is integrated over time and all the sample space:

$$
\begin{aligned}
\int d^{3} r d t\left\langle M^{\alpha}(\boldsymbol{r}, t)\right\rangle_{T} & =\int d^{3} r d t e^{i(0 t-0 \boldsymbol{r})}\left\langle M^{\alpha}(\boldsymbol{r}, t)\right\rangle_{T} \\
& =\left\langle M^{\alpha}(\boldsymbol{Q}=0, \omega=0)\right\rangle_{T} \\
& =\chi^{\prime \alpha \alpha}(\boldsymbol{Q}=0, \omega=0) H^{\alpha} \\
\text { since } \chi^{\prime \prime \alpha \alpha}(\boldsymbol{Q}=0, \omega=0)=0 & =\chi^{\alpha \alpha}(\boldsymbol{Q}=0, \omega=0) H^{\alpha}
\end{aligned}
$$

and hence correponds to the dynamic susceptibility at $Q=0, \omega=0$.

For general $\boldsymbol{Q}, \omega$, the Fourier transformed $\chi^{\alpha \beta}(\boldsymbol{Q}, \omega)=\chi^{\prime \alpha \beta}(\boldsymbol{Q}, \omega)+i \chi^{\prime \prime \alpha \beta}(\boldsymbol{Q}, \omega)$ is complex, and in general $\chi^{\alpha \beta}(\boldsymbol{Q}, \omega)$ is not the same as $\chi^{\beta \alpha *}(\boldsymbol{Q}, \omega)$, i.e. the dynamic susceptibility tensor is not a Hermitian matrix.

\subsection{Diffuse scattering function and dynamic susceptibility: The fluctuation-dissipation theorem}

The "diffuse" part of the scattering function is closely related to the dynamic susceptibility. One can show $[1,3]$ that

$$
\begin{aligned}
S_{\text {diff }}^{\alpha \beta}(\boldsymbol{Q}, \omega) & =S^{\alpha \beta}(\boldsymbol{Q}, \omega)-S_{\text {static }}^{\alpha \beta}(\boldsymbol{Q}, \omega) \\
& =-\frac{\hbar}{\pi} \frac{1}{1-e^{-\frac{\hbar \omega}{k_{B} T}}} \frac{1}{2 i}\left(\chi^{\alpha \beta}(\boldsymbol{Q}, \omega)-\chi^{\beta \alpha *}(\boldsymbol{Q}, \omega)\right)
\end{aligned}
$$




\section{Collection SFN}

$$
\begin{aligned}
S_{\text {diff }}^{\alpha \alpha}(\boldsymbol{Q}, \omega)= & -\frac{\hbar}{\pi} \frac{1}{1-e^{-\frac{\hbar \omega}{k_{B} T}}} \quad \chi^{\prime \prime \alpha \alpha}(\boldsymbol{Q}, \omega) \\
S_{\text {diff }}(\boldsymbol{Q}, \omega)= & -\frac{\hbar}{\pi} \frac{1}{1-e^{-\frac{\hbar \omega}{k_{B} T}}} \\
& \cdot \sum_{\alpha \beta}\left(\delta_{\alpha \beta}-\hat{Q}^{\alpha} \hat{Q}^{\beta}\right) \frac{1}{2}\left(\chi^{\prime \prime \alpha \beta}(\boldsymbol{Q}, \omega)+\chi^{\prime \prime \beta \alpha}(\boldsymbol{Q}, \omega)\right) .
\end{aligned}
$$

These relations are valid independent of the symmetry of the sample. They relate the scattering due to fluctuations to the imaginary part of the susceptibility, and are known as the fluctuation-dissipation theorem.

The second and third relation describe measurable quantities and are real, while the first may be complex. (All observables contain of course real combinations of $S_{\text {diff }}^{\alpha \beta}(\boldsymbol{Q}, \omega)$.)

\subsection{Kramers-Kronig relation}

The Kramers-Kronig relations relate the real part of the complex susceptibility to the imaginary part. An imaginary part determined by neutron scattering allows to reconstruct the whole susceptibility:

$$
\chi^{\prime \alpha \alpha}(\boldsymbol{Q}, \omega)=\frac{1}{\pi} \int d \omega^{\prime} \frac{\chi^{\prime \prime \alpha \alpha}\left(\boldsymbol{Q}, \omega^{\prime}\right)}{\omega^{\prime}-\omega}
$$

In the context of critical scattering the so-called staggered susceptibility is important, which measures the linear answer of a compound at a non-zero wave vector $\boldsymbol{Q}$, and which cannot be measured with a SQUID. Neutron scattering in a finite energy interval and subsequent energy integration allows to obtain this staggered susceptibility via the Kramers-Kronig relation:

$$
\begin{aligned}
\chi^{\prime \alpha \alpha}(\boldsymbol{Q}, \omega=0) & =\frac{1}{\pi} \int_{-\infty}^{\infty} d \omega \frac{\chi^{\prime \prime \alpha \alpha}(\boldsymbol{Q}, \omega)}{\omega-0}=-\int_{-\infty}^{\infty} d \omega \frac{1-e^{-\frac{\hbar \omega}{k_{B} T}}}{\hbar \omega} S_{\text {diff }}^{\alpha \alpha}(\boldsymbol{Q}, \omega) \\
& \approx-\int_{-k_{B} T}^{\omega_{\text {cutoff }}} d \omega \frac{1-e^{-\frac{\hbar \omega}{k_{B} T}}}{\hbar \omega} S_{\text {diff }}^{\alpha \alpha}(\boldsymbol{Q}, \omega)
\end{aligned}
$$

where again the limits $-\infty, \infty$ can be approximated by finite limits, since $S_{d i f f}^{\alpha \alpha}(Q, \omega)$ becomes zero below $-k_{B} T$ and above all magnetic excitation energies $\epsilon_{n}$ of the sample.

The Kramers-Kronig relations also allow to relate the neutron response to the SQUID-susceptibility $\chi^{\prime \alpha \alpha}(\boldsymbol{Q}=0, \omega=0)$ :

$$
\chi^{\alpha \alpha \alpha}(\boldsymbol{Q}=0, \omega=0) \approx-\int_{-k_{B} T}^{\omega_{\text {cutoff }}} d \omega \frac{1-e^{-\frac{\hbar \omega}{k_{B} T}}}{\hbar \omega} S_{d i f f}^{\alpha \alpha}(\boldsymbol{Q}=0, \omega) .
$$




\subsection{Detailed balance}

Generally valid are the following relations for the dynamic susceptibility and the diffuse scattering function [3]:

$$
\begin{aligned}
& \chi^{\alpha \beta}(\boldsymbol{Q}, \omega)=\chi^{\alpha \beta *}(-\boldsymbol{Q},-\omega) \\
& S_{\text {diff }}^{\alpha \beta}(\boldsymbol{Q}, \omega)=S_{\text {diff }}^{\beta \alpha *}(\boldsymbol{Q}, \omega) \\
& S_{\text {diff }}^{\alpha \beta}(\boldsymbol{Q}, \omega)=e^{\frac{\hbar \omega}{k_{B} T}} S_{\text {diff }}^{\beta \alpha}(-\boldsymbol{Q},-\omega), \\
& \underbrace{S_{\text {diff }}(\boldsymbol{Q}, \omega)}_{\text {Detailed balance }}=e^{\frac{\hbar \omega}{k_{B} T}} S_{\text {diff }}(-\boldsymbol{Q},-\omega)
\end{aligned}
$$

where the last equation is obtained by summation $\sum_{\alpha \beta}\left(\delta_{\alpha \beta}-\hat{Q}^{\alpha} \hat{Q}^{\beta}\right)$ on both sides.

In case of centrosymmetry, i.e. for crystals with centrosymmetric structures and for isotropic media (liquids, powders), the following relations hold [3]:

$$
\begin{aligned}
& \chi^{\prime \alpha \beta}(\boldsymbol{Q}, \omega)=\chi^{\prime \alpha \beta}(\boldsymbol{Q},-\omega) \\
& \chi^{\prime \prime \alpha \beta}(\boldsymbol{Q}, \omega)=-\chi^{\prime \prime \alpha \beta}(\boldsymbol{Q},-\omega) \\
& S_{\text {diff }}^{\alpha \beta}(\boldsymbol{Q}, \omega)=e^{\frac{\hbar \omega}{k_{B} T}} S_{\text {diff }}^{\beta \alpha}(+\boldsymbol{Q},-\omega), \\
& \underbrace{\underbrace{}_{\text {diff }}(\boldsymbol{Q}, \omega)=e^{\frac{\hbar \omega}{k_{B} T}} S_{\text {diff }}(\boldsymbol{Q},-\omega)}_{\text {Detailed balance for centrosymmetry }}
\end{aligned}
$$

where again the last equation is obtained by summation $\sum_{\alpha \beta}\left(\delta_{\alpha \beta}-\hat{Q}^{\alpha} \hat{Q}^{\beta}\right)$.

One and the same linear answer of the magnetic material will therefore at different temperatures lead to different scattering functions. Note that the scattering intensity is very different at negative and positive energy transfer at low temperatures - a neutron cannot gain energy from the sample if there are no thermally excited states present, but it can always, even at $T=0$, give energy to the sample and create an excited state.

If one is interested in the temperature dependent changes of the compound's magnetic behaviour, one should always look at the dynamic susceptibility rather than the scattering function, since $S(\boldsymbol{Q}, \omega)$ also depends on the changing occupation number of the excited states. A frequent question addressed by neutron scattering concerns the opening of a gap as the temperature is lowered. $S(\boldsymbol{Q}, \omega)$ at low energies will generally decrease with decreasing $T$ because of the changing occupation number, so that $S(\boldsymbol{Q}, \omega)$ taken at different temperatures quite frequently looks as if there were a gap opening at low temperatures. Only an analysis of the dynamic susceptibility as a function of temperature will correctly answer the question. In the example below, the dynamic susceptibility is temperature independent and gapless (finite at every $\omega>0$ ). There is therefore no gap, neither at low (blue) nor high (red) temperatures. If analysed in terms of a damped harmonic oscillator, the characteristic frequency of the oscillator is zero at both temperatures. 


\section{Collection SFN}

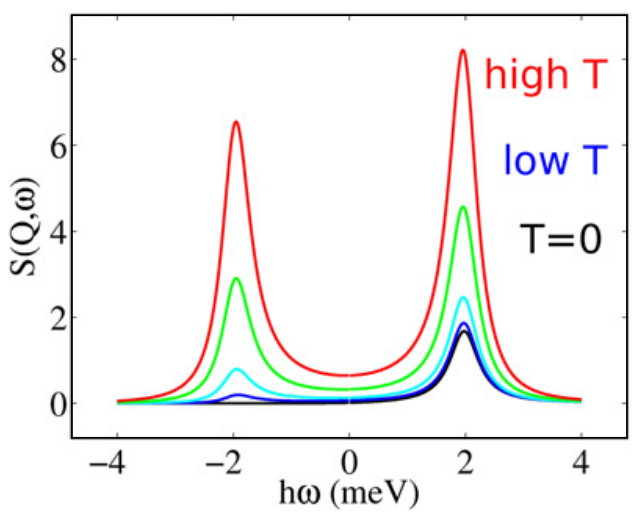

Figure 5. $S(\boldsymbol{Q}, \omega)$ at different temperatures $T$ for a temperature independent dynamic susceptibility $\chi^{\prime \prime}(\boldsymbol{Q}, \omega)$, (increasing $T$ is shown as black-blue-cyan-green-red).
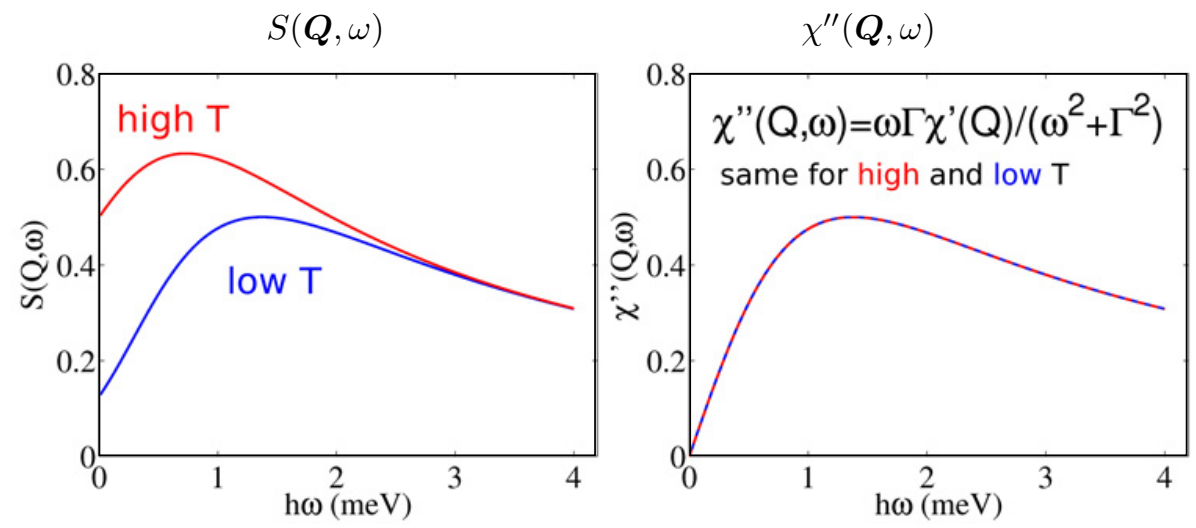

Figure 6. Is there a gap or not at low temperature? - There is no gap! The "shift" of the maximum in $S(\boldsymbol{Q}, \omega)$ from low (blue) to high temperatures (red) can be misleading: The dynamic susceptibility, here chosen as temperature independent, is gapless and finite at every infinitesimally small $\omega>0$, and the characteristic frequency of the harmonic oscillator is zero.

\section{CRYSTALS: "SPIN-ONLY” AND SPIN-LATTICE APPROXIMATIONS}

Important approximations allow to rewrite the neutron scattering cross section not only as Fourier transformed pair correlation of the space and time-dependent magnetization density, but directly as Fourier transform (FT) of the spin pair correlation function, multiplied with form factor and DebyeWaller factor. We first review the conditions of the approximations and then some generalisations, which make this approximation so successful, that, in practice, it is always used [1, 2, 4].

\subsection{Ionic crystals with spin-only moment}

We suppose now

- a ionic crystal where the unpaired electrons are localized around the equilibrium position of the nucleus $\boldsymbol{R}_{n}(t)$ (Heitler-London model)

- LS coupling: $\boldsymbol{S}=\sum_{v} \boldsymbol{s}_{v}$ and $\boldsymbol{L}=\sum_{v} \boldsymbol{\ell}_{v}$ 
- spin-only magnetic moment: $L=0$

- that the neutron energy is too small to change $S$ or $L$ or $\langle\boldsymbol{L}\rangle$.

This means, that the unpaired electrons will have the same spatial wave functions and the same total spin $S$ before and after the scattering process, only the total spin direction $S(t) / S$ and the position of the ion $\boldsymbol{R}_{n}(t)$ (which is also supposed to be the mean position of the unpaired electrons) can change. This means we will average as follows (written very sloppily, see [1] for a thorough treatment):

For a given ion $n$ at $\quad \boldsymbol{R}_{n}(t)$

we have an unpaired electron $v$ at $\quad \boldsymbol{r}(t)=\boldsymbol{R}_{n}(t)+\overline{\boldsymbol{r}_{v}(t)}$

and somewhat more precisely (cf. [1] for a proper derivation) we have for each unpaired electron spin $\boldsymbol{s}_{v}$

$$
\begin{aligned}
\boldsymbol{s}_{v}(\boldsymbol{r}(t), t) & =\boldsymbol{s}_{v}\left(\boldsymbol{R}_{n}(t)+\boldsymbol{r}_{v}(t), t\right) \\
& =\boldsymbol{s}_{v}(t) \delta\left(\boldsymbol{r}-\left(\boldsymbol{R}_{n}(t)+\boldsymbol{r}_{v}(t)\right)\right) \\
& =\boldsymbol{s}_{v}(t) \int d^{3} r^{\prime} \delta\left(\boldsymbol{r}-\boldsymbol{R}_{n}(t)-\boldsymbol{r}^{\prime}\right) \delta\left(\boldsymbol{r}^{\prime}-\boldsymbol{r}_{v}(t)\right) \\
& \approx \boldsymbol{s}_{v}(t) \int d^{3} r^{\prime} \delta\left(\boldsymbol{r}-\boldsymbol{R}_{n}(t)-\boldsymbol{r}^{\prime}\right) \int d^{3} r^{\prime \prime} \delta\left(\boldsymbol{r}^{\prime}-\boldsymbol{r}^{\prime \prime}\right) f_{n}\left(\boldsymbol{r}^{\prime \prime}\right) \\
& =\boldsymbol{s}_{v}(t) \int d^{3} r^{\prime} \delta\left(\boldsymbol{r}-\boldsymbol{R}_{n}(t)-\boldsymbol{r}^{\prime}\right) f_{n}\left(\boldsymbol{r}^{\prime}\right)
\end{aligned}
$$

where $f_{n}(\boldsymbol{r})$ is the probablity to find the unpaired electron at distance $\boldsymbol{r}$ from the ion nucleus, and only depends on the ion $n$ but not on the individual electron $v$ nor on time. We have then

$$
\begin{aligned}
\sum_{v} \boldsymbol{s}_{v}(\boldsymbol{r}(t), t) & \approx \sum_{v} \boldsymbol{s}_{v}(t) \int d^{3} \boldsymbol{r}^{\prime} \delta\left(\boldsymbol{r}-\boldsymbol{R}_{n}(t)-\boldsymbol{r}^{\prime}\right) f_{n}\left(\boldsymbol{r}^{\prime}\right) \\
& =\boldsymbol{S}(t) \int d^{3} r^{\prime} \delta\left(\boldsymbol{r}-\boldsymbol{R}_{n}(t)-\boldsymbol{r}^{\prime}\right) f_{n}\left(\boldsymbol{r}^{\prime}\right) \\
& =\boldsymbol{S}\left(\boldsymbol{R}_{n}(t), \boldsymbol{r}, t\right)
\end{aligned}
$$

where $\boldsymbol{S}\left(\boldsymbol{R}_{n}(t), \boldsymbol{r}, t\right) \cdot \boldsymbol{S}\left(\boldsymbol{R}_{n}(t), \boldsymbol{r}, t\right)=S(S+1)$ is time independent ( $S$ may be different on different sites $n)$.

We can therefore write

$$
\begin{aligned}
\left\langle n_{1} \boldsymbol{k}_{f}|\boldsymbol{M}(\boldsymbol{r})| n_{0} \boldsymbol{k}_{i}\right\rangle & \propto\left\langle n_{1} \boldsymbol{k}_{f}\left|\sum_{v} \boldsymbol{s}_{v}(\boldsymbol{r})\right| n_{0} \boldsymbol{k}_{i}\right\rangle \\
& \approx\left\langle n_{1} \boldsymbol{k}_{f}\left|\boldsymbol{S}\left(\boldsymbol{R}_{n}, \boldsymbol{r}\right)\right| n_{0} \boldsymbol{k}_{i}\right\rangle
\end{aligned}
$$

contract $\boldsymbol{k}_{f}, \boldsymbol{k}_{i}$ and take the Fourier transform, the convolution integral of the spin positions gives a product of Fourier transforms

$$
=\left\langle n_{1}\left|e^{i \boldsymbol{Q R} R_{n}} f_{n}(\boldsymbol{Q}) S\right| n_{0}\right\rangle
$$

and in the cross section, the time-independent $f_{n}(\boldsymbol{Q})$ can be taken out of the matrix element:

$$
\begin{aligned}
& \left|\left\langle n_{1} \boldsymbol{k}_{f}|\boldsymbol{M}(\boldsymbol{r})| n_{0} \boldsymbol{k}_{i}\right\rangle\right|^{2} \delta\left(\epsilon_{1}-\epsilon_{0}-\hbar \omega\right) \\
\propto & \int_{-\infty}^{\infty} d \omega e^{-i \omega t}\left\langle n_{0}\left|e^{-i \boldsymbol{Q} \boldsymbol{R}_{n}(0)} f_{n}(\boldsymbol{Q})^{*} \boldsymbol{S}(0) e^{i \boldsymbol{Q} \boldsymbol{R}_{n}(t)} f_{n}(\boldsymbol{Q}) \boldsymbol{S}(t)\right| n_{0}\right\rangle
\end{aligned}
$$




\section{Collection SFN}

$$
\begin{aligned}
& =\left|f_{n}(\boldsymbol{Q})\right|^{2} \int_{-\infty}^{\infty} d \omega e^{-i \omega t}\left\langle n_{0}\left|e^{-i \boldsymbol{Q} \boldsymbol{R}_{n}(0)} \boldsymbol{S}(0) e^{i \boldsymbol{Q} \boldsymbol{R}_{n}(t)} \boldsymbol{S}(t)\right| n_{0}\right\rangle \\
f_{n}(\boldsymbol{Q}) & =\int d^{3} r f(\boldsymbol{r}) e^{i \boldsymbol{Q} \boldsymbol{r}}
\end{aligned}
$$

is called the magnetic form factor, it is the space-Fourier transform of the unpaired electron shell's spatial wave function. Therefore, for not too large neutron energies we can use the ionic form factor even in the inelastic case.

If we summarize this into a simple picture, the approximations correspond to an unpaired electron shell which is firmly fixed to the nucleus, and where the unpaired electrons are smeared out and completely delocalized, so that their positions are only given by a probability function. In the neutron scattering process the position of the ion can change (but the unpaired electron shell remains firmly attached to it and does not change shape), and the direction of the total electron spin can change, but not its length $S$.

\subsection{Spin only - "extended validity"}

For ionic crystals, even if $L \neq 0$, the Fourier transform of the unpaired electron shell can still be taken outside of the matrix element, under the following conditions:

- quenched orbital momentum:

$$
\begin{aligned}
& \left\langle L^{\alpha}\right\rangle=0 \text { for } \alpha=x, y, z \\
& \boldsymbol{J}=\boldsymbol{S}+\boldsymbol{L}, E_{J} \ll E_{J^{\prime}}
\end{aligned}
$$

- $\quad$ LS-coupling with only one value of $J$ :

- neutron energy small

compared to higher $<L>-, L$ - or $J$-states: $\quad \hbar \omega \ll E_{J^{\prime}}, E_{<L>^{\prime}}$

- $\quad$ small $Q$ :

$$
|\boldsymbol{Q}|^{-1} \gg \text { extension of }
$$

unpaired electron shell.

The table below summarizes how $f(\boldsymbol{Q})$ and $\boldsymbol{S}$ should be replaced in various cases.

\begin{tabular}{l|l|c|c|c} 
Case & Condition (in addition to 3.1) & & & example \\
\hline true $L=0$ & - & $f(\boldsymbol{Q})$ & $\boldsymbol{S}$ & $\mathrm{Mn}^{2+}$ \\
$\langle\boldsymbol{L}\rangle=0$ & small $\boldsymbol{Q}$ & $\frac{g}{2} f(\boldsymbol{Q})$ & $\boldsymbol{S}$ & $\mathrm{Cu}^{2+}$ \\
$\boldsymbol{J}=\boldsymbol{S}+\boldsymbol{L}$ & small $\boldsymbol{Q}, E_{J} \ll E_{J^{\prime} \neq J}$ & $\frac{g_{J}}{2} f(\boldsymbol{Q})$ & $\boldsymbol{J}$ & most but not $\mathrm{Sm}^{3+}, \mathrm{Eu}^{2+}$
\end{tabular}

The transition matrix elements become then

$$
\begin{aligned}
& \left\langle n_{1} \boldsymbol{k}_{f}|\boldsymbol{M}(\boldsymbol{r})| n_{0} \boldsymbol{k}_{i}\right\rangle=\sum_{n} \frac{g_{n}}{2} f_{n}(Q)\left\langle n_{1}\left|e^{i \boldsymbol{Q} \boldsymbol{R}_{n}} \boldsymbol{J}\left(\boldsymbol{R}_{n}\right)\right| n_{0}\right\rangle \\
& \left\langle n_{1} \boldsymbol{k}_{f}|\boldsymbol{M}(\boldsymbol{r})| n_{0} \boldsymbol{k}_{i}\right\rangle=\sum_{n} \frac{g_{n}}{2} f_{n}(\boldsymbol{Q})\left\langle n_{1}\left|e^{i \boldsymbol{Q} \boldsymbol{R}_{n}} S\left(\boldsymbol{R}_{n}\right)\right| n_{0}\right\rangle .
\end{aligned}
$$


JDN 20

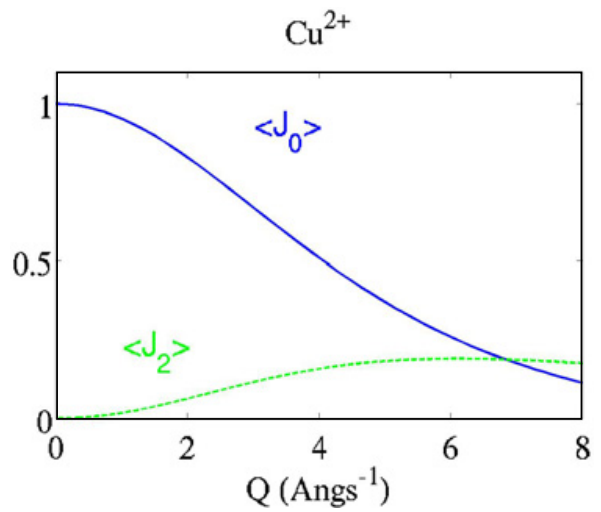

Figure 7. Form factor of the $\mathrm{Cu}^{2+}$ ion, after [6].

If in addition the spatial electron wave function can be approximated as spherically symmetric, the form factor depends on $Q=|Q|$ only:

$$
\begin{aligned}
& \frac{g_{J}}{2} f(Q) \approx \frac{g_{S}}{2} \mathcal{J}_{0}(|Q|)+\frac{g_{L}}{2}\left(\mathcal{J}_{0}(|Q|)+\mathcal{J}_{2}(|Q|)\right) \\
& \mathcal{J}_{n}(|Q|)=4 \pi \int_{0}^{\infty} d r r^{2} \underbrace{j_{n}(|Q| r)}_{\text {spherical Bessel function of order } n} s(r)
\end{aligned}
$$

This approximation, together with the small- $|Q|$ condition, is called the dipolar approximation (cf. [6]). The form factor of the $\mathrm{Cu}^{2+}$ ion [6] is shown above.

\subsection{Spin-lattice decoupling approximation}

In addition to spin-only and dipolar approximation we will now assume that the electron shell spin direction has no effect on the position of the ionic nucleus nor the mean position of the unpaired spin electron cloud (remember that both are firmly coupled in the Heitler-London model). This assumption may not be valid for every aspect in e.g. spin-Peierls compounds and multiferroics. We further assume that the mean position of the unpaired spin electron cloud is the equilibrium position of the ion, and no longer $t$-dependent.

We can then separate the correlation function of the ion positions from the correlation of spin orientations

$$
\left\langle e^{-i \boldsymbol{Q} \boldsymbol{R}_{n}(0)} S^{\alpha}\left(\boldsymbol{R}_{n}(0), 0\right) e^{i \boldsymbol{Q} \boldsymbol{R}_{m}(t)} S^{\beta}\left(\boldsymbol{R}_{m}(t), t\right)\right\rangle_{T} \approx\left\langle e^{-i \boldsymbol{Q} \boldsymbol{R}_{n}(0)} e^{i \boldsymbol{Q} \boldsymbol{R}_{m}(t)}\right\rangle_{T}\left\langle S^{\alpha}\left(\boldsymbol{R}_{n}, 0\right) S^{\beta}\left(\boldsymbol{R}_{m}, t\right)\right\rangle_{T}
$$

and write

$$
\boldsymbol{R}_{n}(t)=\underbrace{\boldsymbol{R}_{n}}_{\text {equilibrium position }}+\underbrace{\boldsymbol{u}_{n}(t)}_{\text {displacement. }}
$$




\section{Collection SFN}

For simplicity we now also assume a Bravais lattice. This makes the formula easier to read, but is not crucial for the approximation.

$$
\begin{aligned}
& \sum_{n m}\left\langle e^{-i \boldsymbol{Q} \boldsymbol{R}_{n}(0)} S^{\alpha}\left(\boldsymbol{R}_{n}(0), 0\right) e^{i \boldsymbol{Q} \boldsymbol{R}_{m}(t)} S^{\beta}\left(\boldsymbol{R}_{m}(t), t\right)\right\rangle_{T} \\
\approx & \sum_{n} e^{i \boldsymbol{Q} \boldsymbol{R}_{n}} \int d t e^{-i \omega t} \underbrace{\left\langle e^{-i \boldsymbol{Q} \boldsymbol{u}(0)} e^{i \boldsymbol{Q} \boldsymbol{\boldsymbol { u } _ { n } ( t )}}\right\rangle_{T}} \underbrace{\left\langle S^{\alpha}(0,0) S^{\beta}\left(\boldsymbol{R}_{n}, t\right)\right\rangle_{T}} \\
= & \sum_{n} e^{i \boldsymbol{Q} \boldsymbol{R}_{n}} \int d t e^{-i \omega t}\left(G_{N}(n, \infty)+G_{N}(n, t)\right)\left(G_{M}^{\alpha \beta}(n, \infty)+G_{M}^{\alpha \beta}(n, t)\right) .
\end{aligned}
$$

At this stage several text books introduce four different types of scattering, according to the four terms that can be formed of the product of the time-independent term and the fluctuation term of the pair correlation functions. $G_{N}(n, t) G_{M}^{\alpha \beta}(n, \infty)$ is typically called magnetovibrational scattering, and $G_{N}(n, t) G_{M}^{\alpha \beta}(n, t)$ is called the fully inelastic term. Note, however, that these terms cannot describe electromagnons nor any kind of correlated spin-lattice excitation, since we arrived at this stage assuming that spin directions and ion positions are uncorrelated. I am not aware of a measurement of these terms, we will simply neglect them in the following. We then remain with

$$
\begin{aligned}
& \approx \sum_{n} e^{i \boldsymbol{Q} \boldsymbol{R}_{n}} \int d t e^{-i \omega t} G_{N}(n, \infty)\left(G_{M}^{\alpha \beta}(n, \infty)+G_{M}^{\alpha \beta}(n, t)\right) \\
& =\left\langle e^{-i \boldsymbol{Q} \boldsymbol{u}}\right\rangle_{T}\left\langle e^{i \boldsymbol{Q} \boldsymbol{u}}\right\rangle_{T} \underbrace{\sum_{n} e^{\boldsymbol{Q} \boldsymbol{R} \boldsymbol{R}_{n}} \int d t e^{-i \omega t}\left(G_{M}^{\alpha \beta}(n, \infty)+G_{M}^{\alpha \beta}(n, t)\right)}_{\left\langle S^{\alpha *}(\boldsymbol{Q}) S^{\beta}(\boldsymbol{Q})\right\rangle_{T, \omega}} \\
& =e^{-2 W(\boldsymbol{Q})}
\end{aligned}
$$

where we have the Debye-Waller factor multiplied with the spin pair correlation function (static and diffuse part). We can imagine the Debye-Waller factor roughly as the Fourier transform of the timeaveraged smeared-out ion position. It decreases with increasing $|\boldsymbol{Q}|$ and increasing $T$ and is essentially 1 in the limit $|\boldsymbol{Q}| \rightarrow 0, T \rightarrow 0$, except for quantum crystals and quantum liquids (like Helium).

\subsection{Unpolarized magnetic neutron cross section}

For a Bravais lattice, the cross section with the previous assumptions reads now:

$$
\begin{gathered}
\text { magnetic "scattering length" } \\
\frac{d^{2} \sigma}{d \Omega d E_{f}}=\frac{\left(\gamma r_{0}\right)^{2}}{2 \pi \hbar} \frac{k_{f}}{k_{i}} \sum_{\alpha \beta}\left(\delta_{\alpha \beta}-\hat{Q}^{\alpha} \hat{Q}^{\beta}\right)\left(\frac{g}{2} f(\boldsymbol{Q})\right)^{2} e^{-2 W(\boldsymbol{Q})} \underbrace{\left\langle S^{\alpha *}(\boldsymbol{Q}) S^{\beta}(\boldsymbol{Q})\right\rangle_{T, \omega}}_{\begin{array}{c}
\text { geometric } \\
\text { selection rule form factor }
\end{array}} \\
\begin{array}{c}
\tilde{S}_{\text {static }}^{\alpha \beta}(\boldsymbol{Q}, \omega=0)+\tilde{S}_{\text {diff }}^{\alpha \beta}(\boldsymbol{Q}, \omega) \\
\text { scattering function }
\end{array}
\end{gathered}
$$

where $\tilde{S}^{\alpha \beta}(\boldsymbol{Q}, \omega)\left(\frac{g}{2} f(\boldsymbol{Q})\right)^{2} e^{-2 W(\boldsymbol{Q})}=S^{\alpha \beta}(\boldsymbol{Q}, \omega)$, i.e. form factor and Debye-Waller factor have been taken out of $S^{\alpha \beta}(\boldsymbol{Q}, \omega)$. 


\subsection{Sum rules}

Important and useful in practice are a number of relations for the zeroth, first and second moment of the scattering function with respect to energy which are known as (frequency or f-) sum rules. Here we consider the zeroth moment using the example of a Bravais ferromagnet in a single domain state with magnetization parallel to $z$.

In a neutron scattering experiment we will find static Bragg as well as "diffuse" scattering, the latter contains magnon creation as well as magnon annihilation terms.

$$
\begin{array}{rlr}
\tilde{S}_{\text {static }}^{z z}(\boldsymbol{Q}, \omega) & =N\left\langle S^{z}\right\rangle_{T}^{2} \frac{(2 \pi)^{3}}{v_{0}} \delta(\omega) \sum_{\tau} \delta(\boldsymbol{Q}-\tau) & \\
\tilde{S}_{\text {diff }}^{y y}(\boldsymbol{Q}, \omega) & =\frac{S}{2} \frac{(2 \pi)^{3}}{v_{0}} \sum_{\tau, \boldsymbol{q}}\left\langle 1+n_{q}\right\rangle_{T} \delta(\boldsymbol{Q}-q-\tau) \delta\left(\hbar \omega-\hbar \omega_{q}\right) \ldots & \text { magnon creation } \\
\ldots+\left\langle n_{\boldsymbol{q}}\right\rangle_{T} \delta(\boldsymbol{Q}+q-\tau) \delta\left(\hbar \omega+\hbar \omega_{q}\right) & \text { magnon annihilation } \\
\hbar \omega_{\boldsymbol{q}} & =2 S J\left(1-\cos \left(q_{a} a\right)\right) & \text { magnon dispersion } \\
n_{\boldsymbol{q}} & =\frac{e^{-\frac{\hbar \omega_{q}}{k_{B} T}}}{1-e^{-\frac{\hbar \omega_{q}}{k_{B} T}}}=\frac{1}{e^{\frac{\hbar \omega_{q}}{k_{B}}}-1} \rightarrow 0 \text { for } T \rightarrow 0 & \text { Bose factor }
\end{array}
$$

Here $\tau$ is a reciprocal lattice vector and $v_{0}$ the volume of the elementary cell.

We notice that the Bragg peak intensity is $N\left\langle S^{z}\right\rangle_{T}^{2}$, while the magnon intensity is $\frac{S}{2}$ for $\tilde{S}^{\alpha \alpha}(\boldsymbol{Q}, \omega)$ with a direction $\alpha=x, y$ orthogonal to the magnetically ordered moment. The Bragg peak is located at the Brillouin zone center, while the magnon exists at all $\mathrm{N} q$-points in the Brillouin zone. For the total integral over the scattering function, integrated over energy and the first Brillouin zone we obtain:

$$
\begin{aligned}
\int d^{3} Q d \omega\left(\tilde{S}_{\text {static }}^{z z}(\boldsymbol{Q}, \omega)+\tilde{S}_{\text {diff }}^{y y}(\boldsymbol{Q}, \omega)+\tilde{S}_{\text {diff }}^{x x}(\boldsymbol{Q}, \omega)\right) & =N \sum_{\alpha}\left\langle\left(S^{\alpha}\right)^{2}\right\rangle_{T} \\
& =N S(S+1)
\end{aligned}
$$

for $T \rightarrow 0=N S^{2}+N S$.

For $T \rightarrow 0$, in a classical ferromagnet, the Bragg peak will hence contain $N S^{2}$, the inelastic scattering (the scattering from magnons) $N S$. As the temperature increases, more and more magnons will be created as well as annihilated in the neutron scattering process, the inelastic intensity will grow, and intensity will be missed in the Bragg peak, while the total sum remains constant. Note that the total sum is independent of the interactions, and therefore a general rule, not only valid for ferromagnets, but also antiferromagnets, short-range ordered and disordered spin systems, paramagnets, and quantum spin magnets that do not order for $T \rightarrow 0$, where hence all scattering is diffuse (inelastic).

\section{POLARIZED NEUTRONS}

This section summarizes the essentials of polarized neutron scattering for inelastic single crystal scattering. Specialized cases (energy integrating or isotropic scattering) are dealt with in other chapters, and more detailed courses can be found in the lecture book of a previous JDN-school devoted to polarized neutron scattering [10]. The neutron spin properties are marked red, sample properties affecting the neutron spin are blue, and sample properties that do not affect the neutron spin are green 


\section{Collection SFN}

in this section. The cross section for a specific transition of the neutron spin state $\sigma_{i} \rightarrow \sigma_{f}$ is

$$
\begin{aligned}
& \qquad \begin{aligned}
\left.\frac{d^{2} \sigma}{d \Omega d E_{f}}\right|_{\sigma_{i} \rightarrow \sigma_{f}} & =\frac{k_{f}}{k_{i}} \sum_{n_{0}} p\left(n_{0}\right)\left|\left\langle n_{1} \sigma_{f}|\tilde{V}(\boldsymbol{Q})| n_{0} \sigma_{i}\right\rangle\right|^{2} \delta\left(\epsilon_{1}-\epsilon_{0}-\hbar \omega\right) \\
\text { where } \quad \tilde{V}(\boldsymbol{Q}) & =\sum_{n} e^{i \boldsymbol{Q} \boldsymbol{R}_{n}}(A_{n}+B_{n} \underbrace{\sigma \cdot \boldsymbol{I}})-\gamma r_{0} \underbrace{\sigma \cdot \boldsymbol{M}_{\perp}(\boldsymbol{Q})}
\end{aligned}
\end{aligned}
$$

is the combined scattering potential. The interaction with the neutron spin has the same form (scalar product) for the nuclear and the electronic spin, and we combine them into the symbol $\mathcal{M}$

$$
\begin{aligned}
\left\langle\sigma_{f} n_{1}|\sigma \cdot \mathcal{M}| n_{0} \sigma_{i}\right\rangle & =\sum_{\alpha}\left\langle\sigma_{f} n_{1}\left|\sigma_{\alpha} \mathcal{M}_{\alpha}\right| n_{0} \sigma_{i}\right\rangle \\
& \approx \sum_{\alpha} \underbrace{\left\langle\sigma_{f}\left|\sigma^{\alpha}\right| \sigma_{i}\right\rangle} \underbrace{\left\langle n_{1}\left|\mathcal{M}_{\alpha}\right| n_{0}\right\rangle} .
\end{aligned}
$$

Dipole matrix elements

For completely uncorrelated nuclear spins we can separate the matrix elements for the neutron spin and the sample, which is extremely useful for e.g. crystal field transitions. We thus obtain the standard dipole transition matrix elements (second line).

Since $\sigma_{z}$ leaves its own eigenstates unchanged, but $\sigma_{x, y}$ changes $\sigma_{z}=+1$ to $\sigma_{z}=-1$ and vice versa, i.e. flips the neutron spin from $+\hat{z}$ to $-\hat{z}$, a useful rule to memorize is

- the neutron spin is not changed by magnetic moments or magnetic eigenvectors (fluctuation amplitudes) that are parallel or antiparallel to it

- the neutron spin is flipped by magnetic moments or magnetic eigenvectors (fluctuation amplitudes) that are orthogonal to it.

Note that this rule is not valid for correlated nuclear spin moments or amplitudes, as in this case the matrix elements for neutron and nuclear spin cannot be separated.

\subsection{Blume-Maleyev equations}

For uncorrelated nuclear spin directions and uncorrelated nuclear isotope distribution, that are not correlated with the electronic magnetization density, the general cross section for polarized neutrons scattering from nuclei and unpaired electrons is written as follows (see [7,8] for the equal-time correlations and [9] for the inelastic scattering in this form):

$$
\begin{aligned}
\frac{d^{2} \sigma}{d \Omega d E_{f}}= & \left\langle N^{*} N\right\rangle_{T, \omega}+\left\langle\boldsymbol{M}_{\perp}^{*} \boldsymbol{M}_{\perp}\right\rangle_{T, \omega}+\left\langle N^{*}\left[\boldsymbol{P}_{i} \cdot \boldsymbol{M}_{\perp}\right]\right\rangle_{T, \omega}+\left\langle N\left[\boldsymbol{P}_{i} \cdot \boldsymbol{M}_{\perp}^{*}\right]\right\rangle_{T, \omega} \\
& +i \boldsymbol{P}_{i} \cdot\left\langle\left[\boldsymbol{M}_{\perp}^{*} \times \boldsymbol{M}_{\perp}\right]\right\rangle_{T, \omega}+\left\langle v_{\mathrm{i}}\right\rangle_{T}+\left\langle\sigma_{\mathrm{nsi}}\right\rangle_{T} \\
\boldsymbol{P}_{f} \frac{d^{2} \sigma}{d \boldsymbol{\Omega} d E_{f}}= & \boldsymbol{P}_{i}\left(\left\langle N^{*} N\right\rangle_{T, \omega}+\left\langle v_{\mathrm{i}}\right\rangle_{T}\right)-\boldsymbol{P}_{i}\left\langle\boldsymbol{M}_{\perp}^{*} \boldsymbol{M}_{\perp}\right\rangle_{T, \omega}-\frac{1}{3} \boldsymbol{P}_{i}\left\langle\sigma_{\mathrm{nsi}}\right\rangle_{T} \\
& +\left\langle\boldsymbol{M}_{\perp}\left[\boldsymbol{P}_{i} \cdot \boldsymbol{M}_{\perp}^{*}\right]+\boldsymbol{M}_{\perp}^{*}\left[\boldsymbol{P}_{i} \cdot \boldsymbol{M}_{\perp}\right]\right\rangle_{T, \omega}-i\left\langle\boldsymbol{M}_{\perp}^{*} \times \boldsymbol{M}_{\perp}\right\rangle_{T, \omega} \\
& +\left\langle N^{*} \boldsymbol{M}_{\perp}+N \boldsymbol{M}_{\perp}^{*}\right\rangle_{T, \omega}-i\left\langle N^{*}\left[\boldsymbol{P}_{i} \times \boldsymbol{M}_{\perp}\right]+i N\left[\boldsymbol{P}_{i} \times \boldsymbol{M}_{\perp}^{*}\right]\right\rangle_{T, \omega}
\end{aligned}
$$


where we have abbreviated

$\begin{array}{ll}\text { nuclear coherent scattering } & N=N(Q) \\ \text { electronic magnetic scattering } & \boldsymbol{M}_{\perp}=\boldsymbol{M}_{\perp}(\boldsymbol{Q}) \\ \text { nuclear spin incoherent scattering } & \sigma_{\mathrm{nsi}} \\ \text { nuclear isotope incoherent } & \nu_{\mathrm{i}} \\ \text { incoming neutron spin polarization } & \boldsymbol{P}_{i} \\ \text { outgoing neutron spin polarization } & \boldsymbol{P}_{f} \\ \text { time Fourier transform and thermal average } & \langle\ldots\rangle_{T, \omega}=\frac{1}{2 \pi} \int_{-\infty}^{\infty} d t e^{-i \omega t}\langle\ldots\rangle_{T} .\end{array}$

For inelastic scattering (with its comparably small intensities), it is practical to consider directly the cross sections for a given incident and outgoing neutron spin state. For diffraction, it can be more practical to consider the polarisation of the outcoming beam as a function of the incoming neutron polarisation (polarisation matrix). We will now go through several common experimental situations for inelastic scattering.

For all of these we define an orthogonal, right-handed coordinate system, with $\hat{\boldsymbol{x}}=\hat{\boldsymbol{Q}}$ and $\boldsymbol{Q}=$ $\boldsymbol{k}_{i}-\boldsymbol{k}_{f}, \hat{z}$ perpendicular to the scattering plane, and $\hat{\boldsymbol{y}}=\hat{z} \times \hat{\boldsymbol{x}}$.

\subsection{Magnetic field in the scattering plane}

In a magnetic field along the scattering wave vector $(\boldsymbol{H} \| \boldsymbol{Q})$, with incoming unpolarized neutrons, the polarisation of the scattered neutrons can be analyzed parallel $(x)$ and antiparallel $(\bar{x})$ to $\boldsymbol{H}\|\boldsymbol{Q}\| \hat{\boldsymbol{x}}$, and the two corresponding cross sections are:

$$
\begin{aligned}
& \left.\frac{d^{2} \sigma}{d \boldsymbol{\Omega} d E_{f}}\right|_{0 \rightarrow x}=\frac{1}{2}\left[\left\langle N^{*} N\right\rangle_{T, \omega}+\left\langle\boldsymbol{M}_{\perp}^{*} \boldsymbol{M}_{\perp}\right\rangle_{T, \omega}+\left\langle v_{\mathrm{i}}\right\rangle_{T}+\left\langle\sigma_{\mathrm{nsi}}\right\rangle_{T}\right]-\frac{i}{2}\left\langle\left[\boldsymbol{M}_{\perp}^{*} \times \boldsymbol{M}_{\perp}\right]\right\rangle_{T, \omega} \cdot \hat{\boldsymbol{x}} \\
& \left.\frac{d^{2} \sigma}{d \boldsymbol{\Omega} d E_{f}}\right|_{0 \rightarrow \bar{x}}=\frac{1}{2}\left[\left\langle N^{*} N\right\rangle_{T, \omega}+\left\langle\boldsymbol{M}_{\perp}^{*} \boldsymbol{M}_{\perp}\right\rangle_{T, \omega}+\left\langle v_{\mathrm{i}}\right\rangle_{T}+\left\langle\sigma_{\mathrm{nsi}}\right\rangle_{T}\right]+\frac{i}{2}\left\langle\left[\boldsymbol{M}_{\perp}^{*} \times \boldsymbol{M}_{\perp}\right]\right\rangle_{T, \omega} \cdot \hat{\boldsymbol{x}} .
\end{aligned}
$$

Subtraction of the two cross sections allows to eliminate background and nuclear scattering and to isolate the chiral term $i\left\langle\left[\boldsymbol{M}_{\perp}^{*} \times \boldsymbol{M}_{\perp}\right]\right\rangle_{T, \omega} \cdot \hat{\boldsymbol{x}}$. The chiral term occurs in form of Bragg peaks in cycloid and helical structures, but also in inelastic neutron scattering, not only for excitations from chiral magnetic structures, but already in ferromagnets. The precession of the spins around the ordering direction $\hat{\boldsymbol{x}}\|\boldsymbol{H}\| \boldsymbol{Q}$ in a ferromagnet can be described by an eigenvector $\boldsymbol{M}_{\perp} \propto(01 i)$. Its vector product with the complex conjugate is as large as the scalar product $\boldsymbol{M}_{\perp}^{*} \cdot \boldsymbol{M}_{\perp}$, so that only one of the two cross sections shows the magnetic excitation. In ferromagnets, where acoustic phonons and magnons emerge both at reciprocal lattice points $Q=\tau$, subtraction of these two cross sections allows to isolate the pure chiral signal of the magnetic excitations. An example is shown below [11].

If the incoming neutrons are polarized parallel $(x)$ or antiparallel $(\bar{x})$ to $\boldsymbol{H}\|\boldsymbol{Q}\| \hat{\boldsymbol{x}}$, and their polarization is analysed as well, the corresponding four cross sections are

$$
\begin{aligned}
& \left.\frac{d^{2} \sigma}{d \Omega d E_{f}}\right|_{x \rightarrow x}=\left.\frac{d^{2} \sigma}{d \Omega d E_{f}}\right|_{\bar{x} \rightarrow \bar{x}}=\left\langle N^{*} N\right\rangle_{T, \omega}+\left\langle\nu_{\mathrm{i}}\right\rangle_{T}+\frac{1}{3}\left\langle\sigma_{\mathrm{nsi}}\right\rangle_{T} \\
& \left.\frac{d^{2} \sigma}{d \Omega d E_{f}}\right|_{x \rightarrow \bar{x}}=\left\langle\boldsymbol{M}_{\perp}^{*} \boldsymbol{M}_{\perp}\right\rangle_{T, \omega}+\frac{2}{3}\left\langle\sigma_{\mathrm{nsi}}\right\rangle_{T}+i\left\langle\left[\boldsymbol{M}_{\perp}^{*} \times \boldsymbol{M}_{\perp}\right]\right\rangle_{T, \omega} \cdot \hat{\boldsymbol{x}} \\
& \left.\frac{d^{2} \sigma}{d \boldsymbol{\Omega} d E_{f}}\right|_{\bar{x} \rightarrow x}=\left\langle\boldsymbol{M}_{\perp}^{*} \boldsymbol{M}_{\perp}\right\rangle_{T, \omega}+\frac{2}{3}\left\langle\sigma_{\mathrm{nsi}}\right\rangle_{T}-i\left\langle\left[\boldsymbol{M}_{\perp}^{*} \times \boldsymbol{M}_{\perp}\right]\right\rangle_{T, \omega} \cdot \hat{\boldsymbol{x}} .
\end{aligned}
$$




\section{Collection SFN}
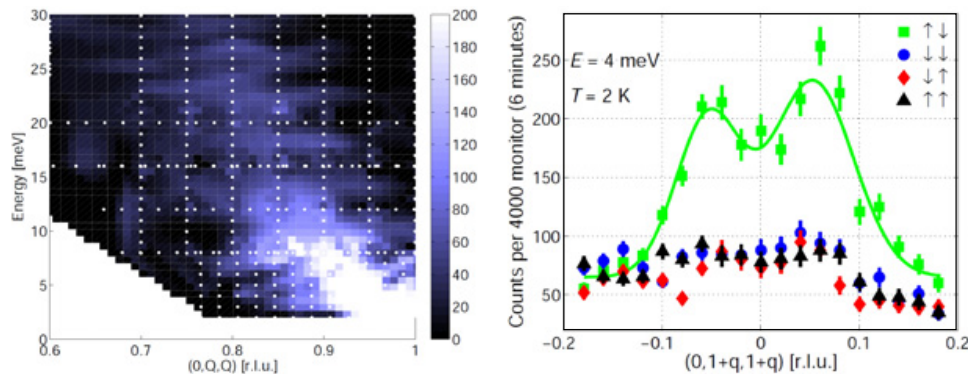

Figure 8. Inelastic chiral term of magnons in a ferromagnet, emerging from a nuclear Bragg peak, measured in a horizontal magnetic field with $\boldsymbol{H} \| \boldsymbol{Q}$. The left side shows the chiral term measured as difference of the two cross sections with incoming unpolarized neutrons, the intensity is color-coded. The right side shows the four cross sections for incoming polarized neutrons and polarisation analysis for one constant-energy scan. The magnon can be seen as a peak in only one of the four cross sections. Measured on IN20, taken from [11].

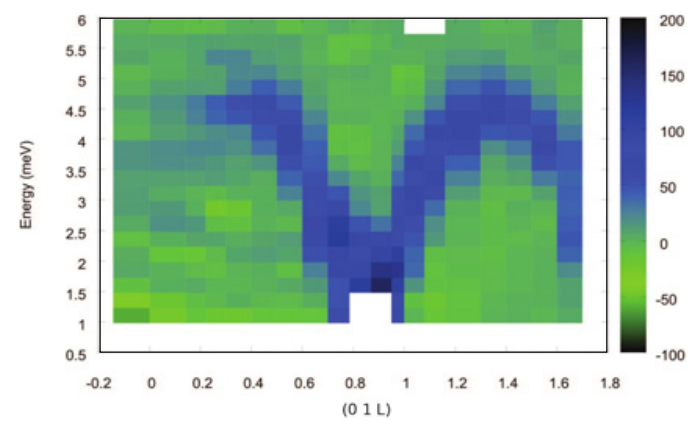

Figure 9. Inelastic chiral term of a mono-domain screw-type magnetic structure (langasite), here measured via $\frac{1}{2}\left(\sigma^{x \bar{x}}-\sigma^{\bar{x} x}\right)$ on IN20, ILL. The intensity is color-coded. Taken from [12].

The inelastic chiral term has also been measured in a langasite, which has a crystal structure without inversion center and orders into a screw-type structure with only one chiral domain [12], see above.

\subsection{Magnetic field perpendicular to the scattering plane}

With polarized incoming neutrons, polarization analysis after the sample and a magnetic field perpendicular to the scattering plane, the initial and final neutron polarization is prepared and measured along the field axis, parallel or antiparallel to the field, $\boldsymbol{P}\|\boldsymbol{H}\| \hat{z}$. Four cross sections can be measured:

$$
\begin{aligned}
& \left.\frac{d^{2} \sigma}{d \Omega d E_{f}}\right|_{\uparrow \uparrow}=\left\langle N^{*} N\right\rangle_{T, \omega}+\left\langle M_{\perp z}^{*} M_{\perp z}\right\rangle_{T, \omega}+\left\langle\nu_{\mathrm{i}}\right\rangle_{T}+\frac{1}{3}\left\langle\sigma_{\text {nis }}\right\rangle_{T}+\left\langle\left[N^{*} M_{\perp z}+N M_{\perp z}^{*}\right]\right\rangle_{T, \omega} \\
& \left.\frac{d^{2} \sigma}{d \Omega d E_{f}}\right|_{\downarrow \downarrow}=\left\langle N^{*} N\right\rangle_{T, \omega}+\left\langle M_{\perp z}^{*} M_{\perp z}\right\rangle_{T, \omega}+\left\langle v_{\mathrm{i}}\right\rangle_{T}+\frac{1}{3}\left\langle\sigma_{\text {nsi }}\right\rangle_{T}-\left\langle\left[N^{*} M_{\perp z}+N M_{\perp z}^{*}\right]\right\rangle_{T, \omega} \\
& \left.\frac{d^{2} \sigma}{d \Omega d E_{f}}\right|_{\uparrow \downarrow}=\left\langle M_{\perp y}^{*} M_{\perp y}\right\rangle_{T, \omega}+\frac{2}{3}\left\langle\sigma_{\text {nsi }}\right\rangle_{T} \\
& \left.\frac{d^{2} \sigma}{d \Omega d E_{f}}\right|_{\downarrow \uparrow}=\left\langle M_{\perp y}^{*} M_{\perp y}\right\rangle_{T, \omega}+\frac{2}{3}\left\langle\sigma_{\text {nsi }}\right\rangle_{T} .
\end{aligned}
$$

This setup is useful for those inelastic cases, where the anisotropy does not allow to keep $\boldsymbol{Q} \| \boldsymbol{H}$ for the $Q$-vectors that one wishes to measure, or if the direction of the applied field matters (e.g. different 
magnetic phases for different field directions with respect to the crystal axes), or if one wishes to separate the magnetic components parallel and perpendicular to the magnetic field. In absence of phonons at the $\boldsymbol{Q}, \omega$ in question, this works generally well, even though a full isolation of the magnetic signal from the incoherent scattering and background is not possible.

For diffraction, the most important application consists in measuring the nuclear-magnetic interference which is directly obtained as the difference of the $\uparrow \uparrow$ and $\downarrow \downarrow$ cross sections. Since the other two cross sections are identical, it is not necessary to analyse the outgoing polarisation:

$$
\begin{aligned}
& \left.\frac{d^{2} \sigma}{d \boldsymbol{\Omega} d E_{f}}\right|_{\uparrow \rightarrow 0}=\left\langle N^{*} N\right\rangle_{T, \omega}+\left\langle\boldsymbol{M}_{\perp}^{*} \cdot \boldsymbol{M}_{\perp}\right\rangle_{T, \omega}+\left\langle v_{\mathrm{i}}\right\rangle_{T}+\left\langle\sigma_{\mathrm{nsi}}\right\rangle_{T}+\left\langle\left[N^{*} M_{\perp z}+N M_{\perp z}^{*}\right]\right\rangle_{T, \omega} \\
& \left.\frac{d^{2} \sigma}{d \Omega d E_{f}}\right|_{\downarrow \rightarrow 0}=\left\langle N^{*} N\right\rangle_{T, \omega}+\left\langle\boldsymbol{M}_{\perp}^{*} \cdot \boldsymbol{M}_{\perp}\right\rangle_{T, \omega}+\left\langle v_{\mathrm{i}}\right\rangle_{T}+\left\langle\sigma_{\mathrm{nsi}}\right\rangle_{T}-\left\langle\left[N^{*} M_{\perp z}+N M_{\perp z}^{*}\right]\right\rangle_{T, \omega}
\end{aligned}
$$

and the interference term is obtained as the difference of the two cross sections with opposite incoming polarisation.

\subsection{Polarization analysis along three perpendicular directions}

A very powerful method consists in using polarized incident neutrons and polarisation analysis after scattering at the sample, and measuring the cross sections for three orthogonal axes of the neutron polarisation. The axis of the neutron polarization at the sample can be defined by a weak magnetic field (1-6 mT) which for many cases will be equivalent to zero field (Helmholtz setup), or alternatively be prepared field-free (Cryopad, Mupad). This method is sometimes called longitudinal polarization analysis or diagonal polarization analysis in order to indicate that the quantization axis for the neutron spin is the same for the incident and the scattered beam. If we define as before a righthanded spin coordinate system $\hat{\boldsymbol{x}} \| \boldsymbol{Q}=\boldsymbol{k}_{i}-\boldsymbol{k}_{f}, \hat{z}$ vertical, $\hat{\boldsymbol{y}}=\hat{\boldsymbol{z}} \times \hat{\boldsymbol{x}}$, and abbreviate the differential cross section $\left.\frac{d^{2} \sigma}{d \Omega d E_{f}}\right|_{\alpha \beta}$ as $\sigma^{\alpha \beta}$, we have the following six equations

$$
\begin{aligned}
& \sigma^{x x}=\sigma^{\overline{x x}}=\left\langle N^{*} N\right\rangle_{T, \omega}+\left\langle v_{\mathrm{i}}\right\rangle_{T}+\frac{1}{3}\left\langle\sigma_{\text {nsi }}\right\rangle_{T} \\
& \frac{1}{2} \sigma^{x \bar{x}}+\frac{1}{2} \sigma^{\bar{x} x}=\quad+\left\langle M_{\perp y}^{*} M_{\perp y}\right\rangle_{T, \omega}+\left\langle M_{\perp z}^{*} M_{\perp z}\right\rangle_{T, \omega}+\frac{2}{3}\left\langle\sigma_{\mathrm{nsi}}\right\rangle_{T} \\
& \frac{1}{2} \sigma^{y y}+\frac{1}{2} \sigma^{\overline{y y}}=\left\langle N^{*} N\right\rangle_{T, \omega}+\left\langle v_{\mathrm{i}}\right\rangle_{T}+\left\langle M_{\perp y}^{*} M_{\perp y}\right\rangle_{T, \omega} \quad+\frac{1}{3}\left\langle\sigma_{\text {nsi }}\right\rangle_{T} \\
& \sigma^{y \bar{y}}=\sigma^{\bar{y} y}=\quad+\left\langle M_{\perp z}^{*} M_{\perp z}\right\rangle_{T, \omega}+\frac{2}{3}\left\langle\sigma_{\mathrm{nsi}}\right\rangle_{T} \\
& \frac{1}{2} \sigma^{z z}+\frac{1}{2} \sigma^{\overline{z z}}=\left\langle N^{*} N\right\rangle_{T, \omega}+\left\langle v_{\mathrm{i}}\right\rangle_{T} \quad+\left\langle M_{\perp z}^{*} M_{\perp z}\right\rangle_{T, \omega}+\frac{1}{3}\left\langle\sigma_{\mathrm{nsi}}\right\rangle_{T} \\
& \sigma^{z \bar{z}}=\sigma^{\bar{z} z}=\quad+\left\langle M_{\perp y}^{*} M_{\perp y}\right\rangle_{T, \omega} \quad+\frac{2}{3}\left\langle\sigma_{\text {nsi }}\right\rangle_{T}
\end{aligned}
$$

We can now isolate the purely magnetic scattering either from the "non-spin flip" cross sections, where the neutron spin remains unchanged in the scattering process, or from the "spin-flip" cross sections, where the neutron spin is reversed in the scattering process. The latter is statistically favorable in presence of phonons in the spectrum, the former is favorable, if not phonons, but rather the (nuclear spin)- incoherent background is the dominant nuisance:

$$
\begin{array}{ll}
\left\langle\boldsymbol{M}_{\perp}^{*} \boldsymbol{M}_{\perp}\right\rangle_{T, \omega}=\sigma^{x \bar{x}}+\sigma^{\bar{x} x} & -\sigma^{y \bar{y}}-\sigma^{z \bar{z}} \\
\left\langle\boldsymbol{M}_{\perp}^{*} \boldsymbol{M}_{\perp}\right\rangle_{T, \omega} & =\frac{1}{2} \sigma^{y y}+\frac{1}{2} \sigma^{\overline{y y}}+\frac{1}{2} \sigma^{z z}+\frac{1}{2} \sigma^{\overline{z z}}-2 \sigma^{x x} .
\end{array}
$$




\section{Collection SFN}

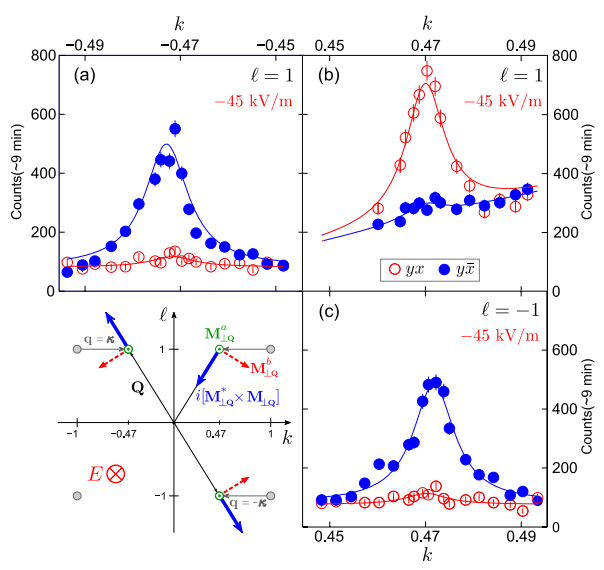

Figure 10. Measurement of the rotation sense of a cycloid magnetic structure in an electric field. The figures display the cross sections $\sigma^{y x}$ (open red circles) and $\sigma^{y \bar{x}}$ (filled blue circles) measured on IN20 with orthogonal incoming $(\| \hat{\boldsymbol{y}})$ and outgoing neutron polarization $(\| \pm \hat{\boldsymbol{x}})$. Taken from [13].

We can also separate different directions of the magnetic moments or fluctuation amplitudes (eigenvectors of excitations), the chiral term, the pure nuclear-spin incoherent scattering, or (the real part of) the nuclear magnetic interference terms, provided the sample is in a single-domain state:

$$
\begin{array}{r}
\left\langle M_{\perp y}^{*} M_{\perp y}\right\rangle_{T, \omega}=\frac{1}{2} \sigma^{y y}+\frac{1}{2} \sigma^{\overline{y y}}-\sigma^{x x} \\
=\frac{1}{2} \sigma^{x \bar{x}}+\frac{1}{2} \sigma^{\bar{x} x}-\sigma^{y \bar{y}} \\
\left\langle M_{\perp z}^{*} M_{\perp z}\right\rangle_{T, \omega}=\frac{1}{2} \sigma^{z z}+\frac{1}{2} \sigma^{\overline{z z}}-\sigma^{x x} \\
=\frac{1}{2} \sigma^{x \bar{x}}+\frac{1}{2} \sigma^{\bar{x} x}-\sigma^{z \bar{z}} \\
\frac{4}{3}\left\langle\sigma_{\mathrm{nsi}}\right\rangle_{T}=2 \sigma^{y \bar{y}}+2 \sigma^{z \bar{z}}-\left(\sigma^{x \bar{x}}+\sigma^{\bar{x} x}\right) \\
2 i \hat{\boldsymbol{x}} \cdot\left\langle\left[\boldsymbol{M}_{\perp}^{*} \times \boldsymbol{M}_{\perp}\right]\right\rangle_{T, \omega}=\sigma^{x \bar{x}}-\sigma^{\bar{x} x} \\
2\left\langle N^{*} M_{\perp y}+N M_{\perp y}^{*}\right\rangle_{T, \omega}=\sigma^{y y}-\sigma^{\overline{y y}} \\
2\left\langle N^{*} M_{\perp z}+N M_{\perp z}^{*}\right\rangle_{T, \omega}=\sigma^{z z}-\sigma^{\overline{z z}} .
\end{array}
$$

For weak magnetic signals, the separation of the magnetic signal from both phonons and incoherent signals is essential. Measurement of just $\sigma^{x \bar{x}}$ leads to erroneous results, since the incoherent nuclear spin scattering frequently displays an energy dependence which follows the density of states of all excitations in the sample, due to multiple scattering processes in the sample (incoherent plus inelastic). 
It is further possible to analyse the final neutron polarisation along an arbitrary direction, even perpendicular to the initial neutron polarisation. For general $\boldsymbol{Q}, \omega$ this can be achieved in a wellcontrolled manner, if the sample is in a strict zero-field environment. Such an experimental setup allows to determine two additional correlations (the imaginary parts of the nuclear-magnetic interference terms), which are important for the analysis of complex magnetic structures, cf. chapter of E. Ressouche.

Below we show an example of a cycloid ferroelectric where the incoming neutron polarization was prepared along $\hat{\boldsymbol{y}}$ and the outgoing neutron polarization measured along $\pm \hat{\boldsymbol{x}}$. The rotation sense of the cycloid magnetic structure was commanded by an electric field [13] and could also be switched (not shown here). In this example the chiral term has nearly the same size as the scalar product $\boldsymbol{M}_{\perp}^{*} \boldsymbol{M}_{\perp}$ so that for each of the three wave vectors a peak appears in only one of the two cross sections $\sigma^{y x}, \sigma^{y \bar{x}}$.

\section{CONCLUSION}

Neutron scattering is a unique technique for studies of magnetism, since it probes directly the Fourier transform of the two-spin correlation function, or more generally, the magnetic pair correlations. Due to the weakness of the neutron-matter interaction, there is normally only one scattering process for each neutron, and the correlation functions are probed in the whole volume. (In large samples, and for strong scattering (Bragg peaks), as well as when searching for exceedingly small signals, multiple scattering nevertheless needs to be taken into acount.) Neutrons are sensitive to electronic magnetic moments or fluctuation amplitudes perpendicular to the momentum transfer. Elastic scattering $(\omega=0)$ reflects the $t \rightarrow \infty$ correlations (Bragg peaks, short range order, glassy frozen states). Diffraction (energy integrated detection) is proportional to the equal-time correlations $(t=0)$. Inelastic scattering reflects the correlated fluctuations, and is directly related to the dynamic susceptibility. Finally, polarised neutrons allow to measure the magnetic signal, its individual components (and more), and to isolate them from other contributions as well as from background.

\section{References}

[1] G.L. Squires, Introduction to the Theory of Neutron Scattering, Dover Publications, 1996.

[2] P.G. de Gennes, Theory of Neutron Scattering by Magnetic Crystals, in Magnetism, Vol. III, Eds. G.T. Rado and H. Suhl (New York: Academic Press, 1963), p.115-147.

[3] W. Marshall and R.D. Lowde, Magnetic Correlations and Neutron Scattering, Rep. Progr. Phys. 31, 705 (1968).

[4] S.W. Lovesey, Theory of Neutron Scattering from Condensed Matter, Vol.2: Polarization effects and Magnetic Scattering, Clarendon Press, Oxford 1986.

[5] Neutron Scattering, ed. by K. Sköld, D.L. Price, Methods in Experimental Physics, vol. 23C (Academic, New York, 1987).

[6] Jane Brown, http://www.ill.eu/sites/ccsl/ffacts/ffachtml.html.

[7] M. Blume, Phys. Rev. 130, 1670 (1963).

[8] S.V. Maleyev, V.G. Bar’yakhtar, R.A. Suris, Sov. Phys. -Solid State 4, 2533 (1963).

[9] S.V. Maleyev, Polarized Neutron Scattering in Magnets, Physics - Uspekhi 45, 569-596 (2002).

[10] Ecole thématique de la SFN, Neutrons Polarisés, ed. by N. Kernavanois, E. Ressouche, H. Schober, J.L. Soubeyroux, EDP Sciences 2005.

[11] R.A. Ewings et al., Phys. Rev. B 82, 144401 (2010).

[12] M. Loire et al., Phys. Rev. Lett. 106, 207201 (2011).

[13] M. Mourigal et al., Phys. Rev. B 83, 100409 (2011).

[14] A.T. Boothroyd, 6th PSI Summer School on Condensed Matter Research, Zuoz, Aug 18-25, 2007; http://xray.physics.ox.ac.uk/boothroyd/Boothroyd\%20Zuoz\%20007.pdf. 